\title{
$P$-wave dispersion and attenuation due to scattering by aligned fluid saturated fractures with finite thickness: theory and experiment
}

\author{
Junxin Guo, ${ }^{1,2}$ Da Shuai, ${ }^{3}$ Jianxin Wei, ${ }^{3}$ Pinbo Ding ${ }^{3}$ and Boris Gurevich ${ }^{2,4}$ \\ ${ }^{1}$ Department of Earth and Space Sciences, Southern University of Science and Technology, Shenzhen 518055, China \\ ${ }^{2}$ WA School of Mines: Minerals, Energy and Chemical Engineering, Curtin University, GPO Box U1987, Perth 6845, Western Australia, Australia \\ ${ }^{3}$ State Key Laboratory of Petroleum Resources and Prospecting and CNPC Key Laboratory of Geophysical Exploration, China University of Petroleum, \\ Beijing 102249, China.E-mail: cup.shuaida@qq.com \\ ${ }^{4}$ CSIRO, 26 Dick Perry Avenue, Kensington 6152, Western Australia, Australia
}

Accepted 2018 September 27. Received 2018 September 22; in original form 2017 November 10

\begin{abstract}
SUMMAR Y
Fractures often play an important role in controlling the fluid flow in hydrocarbon reservoirs. When the seismic wave propagates through media containing fracture corridors, significant scattering dispersion and attenuation can occur. In this work, we study the $P$-wave dispersion and attenuation due to the scattering caused by 2-D fluid-saturated aligned fractures with finite thickness, which are embedded in an isotropic elastic background medium. Using the Foldy approximation and the representation theorem, the $P$-wave dispersion and attenuation are related to the displacement discontinuities across the fractures. These fracture displacement discontinuities are obtained from the boundary conditions and the $P$-wave dispersion and attenuation can thus be calculated. A numerical example shows that the fracture thickness has significant influence on the dispersion and attenuation, especially in the low-frequency regime when the fracture size is smaller than the seismic wavelength. The effects of the fluid bulk modulus are also significant, which are opposite to those of the fracture thickness. However, the effect of the fluid viscosity is found to be negligible for the studied configurations. To validate the proposed model, the theoretical predictions are compared with ultrasonic measurements on fractured samples. The comparison shows overall good agreement between theory and experiment. This work reveals the important influence of fracture thickness and saturating fluid properties on the $P$-wave scattering dispersion and attenuation. Hence, it shows a potential to extract these parameters from seismic data.
\end{abstract}

Key words: Seismic attenuation; Theoretical seismology; Wave propagation; Wave scattering and diffraction; Fractures, faults, and high strain deformation zones.

\section{INTRODUCTION}

Naturally fractured reservoirs constitute substantial part of oil and gas reserves worldwide. Importance of fractures was probably first understood in the context of carbonate reservoirs. In recent years, there has been a renewed interest in fractured unconventional reservoirs such as tight gas sands, gas shales and coal. Understanding of fractures is important as they play an important role in controlling the fluid flow. In some reservoirs, so-called sweet spots (areas of intense fracturing) are the only areas where economic production is possible. Of particular importance for production are fracture 'swarms' or 'corridors', which are large zones of densely spaced fractures tens of metres in height and several hundred metres in length, up to several metres in width and with permeability on the order of several Darcies (Bush 2010). Hence, it is important to detect and characterize the fracture corridors before drilling many wells. To this end, the seismic method presents a special value due to its non-invasive nature and a large detection scale. As the size of fractures in such fractured zones is often comparable or even larger than the seismic wavelength, the wave scattering by fractures can be significant (e.g. Wu \& Aki 1985; Gurevich et al. 1997; Vlastos et al. 2003, 2006, 2007; Sato et al. 2011). This can result in substantial dispersion and attenuation of seismic waves, which in turn can serve as potential seismic attributes for the detection and characterization of fractured reservoirs (Vasconcelos \& Jenner 2005; Burns et al. 2007). For this purpose, it is essential to understand and quantify the mechanism of the dispersion and attenuation due to the scattering by fractures.

Scattering of elastic waves by fractures have been considered previously in the context of both geophysics and non-destructive testing of materials. Dispersion and attenuation due to wave scattering by a single dry fracture have been considered by a number of authors (e.g. Mal 1970a,b; Martin 1981; Krenk \& Schmidt 1982; Keogh 1986; Martin \& Rizzo 1989). Most of these studies investigate interactions of 
the elastic waves with a 2-D (slit) or 3-D (penny-shaped) fracture in an infinite isotropic elastic solid. Based on these theoretical models, the effects of multiple dry fractures can be studied using the non-interaction approximation proposed by Foldy (1945). This approach has been used to compute wave scattering in an elastic solid containing a random distribution of fractures (e.g. Kikuchi 1981; Zhang \& Achenbach 1991; Kawahara 1992; Kawahara \& Yamashita 1992; Zhang \& Gross 1993a,b). Besides the Foldy approximation, other approaches have also been used to take into account the effects of fracture interactions and non-flat fractures (e.g. Sabina et al., 1993; Smyshlyaev \& Willis 1993a,b; Murai et al. 1995; Murai 2007; Caleap et al. 2009). Some of these approaches have been validated by numerical simulations (e.g. Suzuki et al. 2006, 2013).

The above studies of scattering have been done for dry fractures. Yet, fractures in hydrocarbon reservoirs and aquifers are important conduits for the fluid flow and hence are often saturated with fluid (e.g. Bush 2010). Therefore, it is essential to model wave scattering by the fluid saturated fractures. To the authors' knowledge, however, only a few studies have been published on this aspect. Kawahara \& Yamashita (1992) modelled the scattering by aligned fluid saturated fractures, which focused on the effects of viscous friction of the fluid on wave dispersion and attenuation. That study was then extended by Murai et al. (1995) by considering fracture interactions. Sabina et al. (1993) and Smyshlyaev \& Willis (1993a,b) applied a dynamic self-consistent approach to wave propagation in an elastic solid with a random distribution of penny-shaped fluid saturated fractures. This problem was also studied by Eriksson et al. (1995) using the T-matrix and Foldy approximations.

Most of the studies mentioned above consider the effects of fractures of infinitesimal thickness. For dry fractures both the effective quasi-static moduli and scattering are controlled by the fracture density, while the influence of the fracture thickness is negligible, provided that the fracture aspect ratio is not very large (usually smaller than 0.1) (e.g. Schoenberg \& Douma 1988; Sabina et al. 1993; Smyshlyaev \& Willis 1993b). However, for fluid saturated fractures, the fracture thickness has a significant influence on the elastic properties of the fractured rocks and therefore, on wave propagation even in the quasi-static regime (e.g. Kuster \& Toksöz 1974; Guo et al. 2018a, 2018b). Hence, in the dynamic regime, it is also essential to study the effects of the fracture thickness on wave scattering and the corresponding dispersion and attenuation for the case of fluid saturated fractures.

In this paper, we develop a model for the $P$-wave scattering dispersion and attenuation by aligned fluid saturated 2-D fractures with finite thickness, which are distributed randomly in an isotropic elastic background medium. The model is developed by extending the model for dry open fractures proposed by Kawahara (1992). A numerical example is used to explore the effects of the fracture thickness and the saturating fluid properties on wave scattering dispersion and attenuation. To validate this model, the theoretical predictions are also compared to the ultrasonic measurements on fractured samples.

\section{THEORETICAL BACKGROUND}

The $P$-wave scattering model for fluid saturated fractures can be developed based on the model for dry open slit fractures, which was proposed by Kawahara (1992). In Kawahara's model, aligned 2-D dry slit fractures are assumed to be distributed randomly and sparsely in an infinite elastic (non-porous) isotropic background medium, as shown in Fig. 1. The coordinate system is chosen such that the fracture plane is perpendicular to the $X_{2}$-axis, and the fracture length along $X_{3}$-axis is infinite. Hence, the plane strain condition in $X_{1}-X_{2}$ plane is satisfied and the 3-D problem can be reduced to a 2-D problem. It is assumed that all the fractures have an identical rectangular shape with a thickness $\beta$ along $X_{2}$-axis and a length $2 a$ along $X_{1}$-axis. The number density of the fractures (number of fractures per unit area) is $v$. We consider a longitudinal plane wave ( $P$ wave) propagating in the fractured rock at an incidence angle of $\theta$ with respect to the fracture normal ( $X_{2}$-axis).

The incident $P$-wave is assumed to be a plane time-harmonic wave with an angular frequency $\omega$ and displacement

$\mathbf{u}_{\mathbf{A}}^{0}=A_{0} e^{i k_{\mathrm{p}} X_{1} \sin \theta+i k_{\mathrm{p}} X_{2} \cos \theta}(\sin \theta, \cos \theta)$,

where $A_{0}$ is the displacement amplitude, $k_{\mathrm{p}}=\omega / V_{\mathrm{p}}$ the wave number, and $V_{\mathrm{p}}$ the $P$-wave velocity in the background medium (for brevity, the time factor $e^{-i \omega t}$ is omitted).

Due to the scattering of the randomly distributed fractures, the total wavefield $\mathbf{u}_{\mathbf{A}}$ is the sum of the incident wave plus the scattered wave. The ensemble average of this total field is called the mean field $\left\langle\mathbf{u}_{\mathbf{A}}\right\rangle$ and has the following form (Kawahara 1992):

$\left\langle\mathbf{u}_{\mathbf{A}}\right\rangle=A e^{i k_{\mathrm{p}} X_{1} \sin \theta+i\left(k_{\mathrm{p}} \cos \theta+\kappa\right) X_{2}}\left(\sin \theta, \cos \theta+\kappa / k_{\mathrm{p}}\right)$,

where $A$ is the initial displacement amplitude of the mean wavefield, and $\kappa$ is the coefficient that determines the attenuation and dispersion of the mean wave.

Since the fractures are distributed sparsely and randomly in the solid, the relationship between the incident wavefield and the ensemble averaging can be replaced by spatial averaging using the Foldy (1945) approximation as follows:

$\left\langle\mathbf{u}_{\mathbf{A}}\right\rangle=\mathbf{u}_{\mathbf{A}}^{0}+v \int \mathbf{S}_{\mathbf{A}}\left\langle\mathbf{u}_{\mathbf{i}}\right\rangle d \mathbf{r}_{\mathbf{i}}$

where $\mathbf{S}_{\mathbf{A}}\left\langle\mathbf{u}_{\mathbf{i}}\right\rangle$ is the scattered wave by the $i$ th fracture due to the incidence of the mean wave $\left\langle\mathbf{u}_{\mathbf{i}}\right\rangle ; \mathbf{r}_{\mathbf{i}}$ denotes the central location of the $i$ th fracture. By integrating over the entire volume of the fractured rock, the total scattered displacement is obtained, which is added to the incident displacement field to obtain the displacement field of the mean wave. 


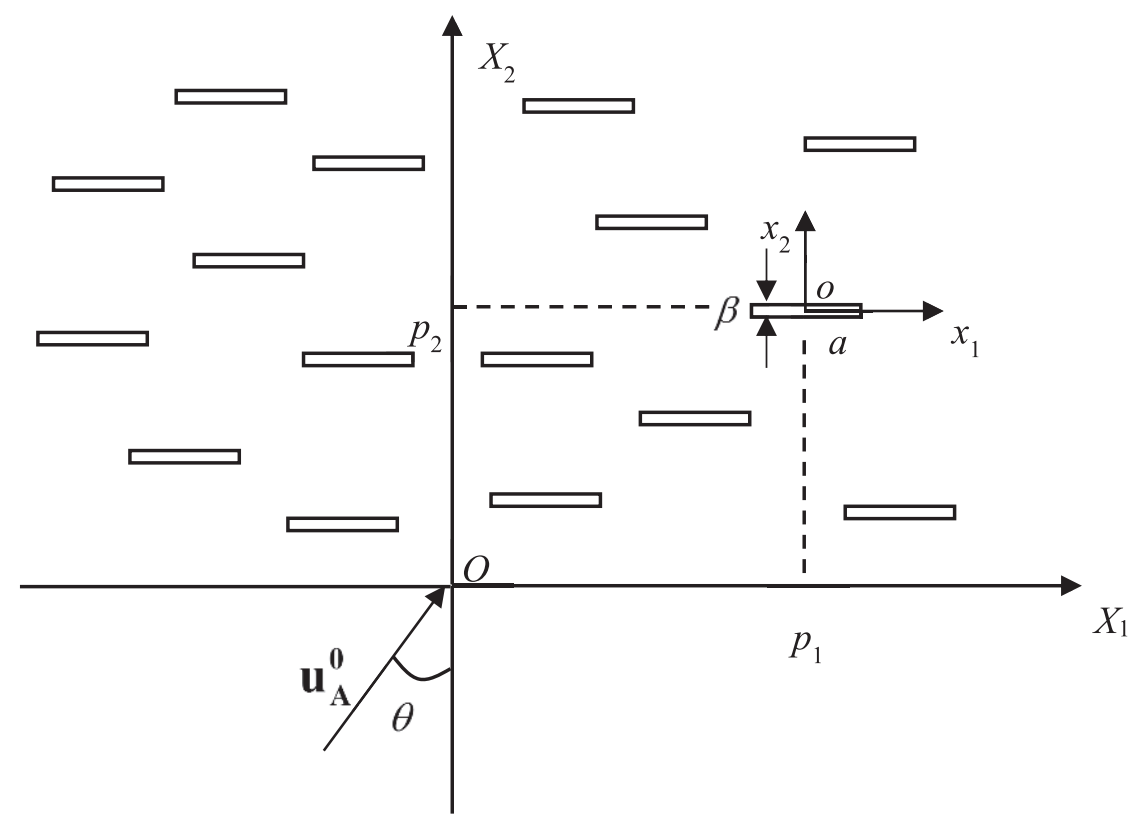

Figure 1. Infinite elastic background medium embedded with randomly and sparsely distributed aligned 2-D slit fractures. The length and thickness of the fracture is $2 a$ and $\beta$, respectively. Both the global and local coordinate systems are established with the $X_{1}$ - (or $x_{1}-$ ) and $X_{2}$ - (or $x_{2}$-) parallel and perpendicular to the fracture plane, respectively. The origin of the local coordinate system is located at the centre of the $i$ th fracture with a global coordinate $\left(p_{1}, p_{2}\right)$.

From eq. (2), the mean incident wave $\left\langle\mathbf{u}_{\mathbf{i}}\right\rangle$ on the $i$ th fracture can be written as

$\left\langle\mathbf{u}_{\mathbf{i}}\right\rangle=A e^{i k_{\mathrm{p}}\left(x_{1}+p_{1}\right) \sin \theta+i\left(k_{\mathrm{p}} \cos \theta+\kappa\right)\left(x_{2}+p_{2}\right)}\left(\sin \theta, \cos \theta+\kappa / k_{\mathrm{p}}\right)$,

where $\left(p_{1}, p_{2}\right)$ is the location of the centre of the $i$ th fracture in the global coordinate system $\left(X_{1}, X_{2}\right) .\left(x_{1}, x_{2}\right)$ defines the location in the local coordinate system with the origin at the centre of the $i$ th fracture and the $x_{1}$-axis and $x_{2}$-axis parallel and perpendicular to the fracture plane, respectively (Fig. 1).

Using the representation theorem, we can present the wavefield scattering by the $i$ th fracture as (e.g. Achenbach 1973; Kawahara \& Yamashita 1992)

$\left[\mathbf{S}_{\mathbf{i}}\left\langle\mathbf{u}_{\mathbf{i}}\right\rangle\right]_{j}=-\int_{-a}^{a}\left[\Delta \mathbf{u}_{\mathbf{i}}\left(\zeta_{1}, p_{1}, p_{2}\right)\right]_{l} \Gamma_{j l}\left(x_{1}, x_{2} \mid \zeta_{1}, 0\right) d \zeta_{1}, \quad j, l=1,2$,

where $[\cdot]_{j}$ represents the $j$ th component of the vector field, the summation convention is employed for the repeated subscripts on the right-hand side of the equation, $\Delta \mathbf{u}_{\mathbf{i}}$ is the displacement discontinuity across the fracture while $\Gamma_{j l}$ has the form

$$
\begin{aligned}
\Gamma_{j l}\left(x_{1}, x_{2} \mid \zeta_{1}, \zeta_{2}\right)= & \frac{i}{4}\left[\delta_{l 2}\left(1-2 \frac{k_{\mathrm{p}}^{2}}{k_{\mathrm{s}}^{2}}\right) \frac{\partial}{\partial x_{j}} H_{0}^{(1)}\left(k_{\mathrm{p}} R\right)+\left(\delta_{j l} \frac{\partial}{\partial x_{2}}+\delta_{j 2} \frac{\partial}{\partial x_{l}}\right) H_{0}^{(1)}\left(k_{\mathrm{s}} R\right)\right. \\
& \left.-\frac{2}{k_{\mathrm{s}}^{2}} \frac{\partial^{3}}{\partial x_{j} \partial x_{l} \partial x_{2}}\left(H_{0}^{(1)}\left(k_{\mathrm{p}} R\right)-H_{0}^{(1)}\left(k_{\mathrm{s}} R\right)\right)\right], j, l=1,2,
\end{aligned}
$$

where $k_{\mathrm{s}}=\omega / V_{\mathrm{s}}$ is the wavenumber of the $S$ wave in the background medium, $V_{\mathrm{s}}$ is its velocity, $\delta_{j l}$ is the Kronecker's delta; $H_{0}^{(1)}(\cdot)$ is the zeroth-order Hankel function of the first kind, and $R$ is given by

$R^{2}=\left(x_{1}-\zeta_{1}\right)^{2}+\left(x_{2}-\zeta_{2}\right)^{2}$.

According to Hooke's law for the isotropic elastic medium (Timoshenko \& Goodier 1934), the stress field induced by $\left\langle\mathbf{u}_{\mathbf{i}}\right\rangle$ and $\mathbf{S}_{\mathbf{i}}\left\langle\mathbf{u}_{\mathbf{i}}\right\rangle$ can be represented as follows (Kawahara \& Yamashita 1992):

$\sigma_{j k}^{E}=\lambda \delta_{j k} \frac{\partial}{\partial x_{l}}\left[\left\langle\mathbf{u}_{\mathbf{i}}\right\rangle\right]_{l}+\mu\left(\frac{\partial}{\partial x_{k}}\left[\left\langle\mathbf{u}_{\mathbf{i}}\right\rangle\right]_{j}+\frac{\partial}{\partial x_{j}}\left[\left\langle\mathbf{u}_{\mathbf{i}}\right\rangle\right]_{k}\right), j, k, l=1,2$,

$\sigma_{j k}^{S}=\lambda \delta_{j k} \frac{\partial}{\partial x_{l}}\left[\mathbf{S}_{\mathbf{i}}\left\langle\mathbf{u}_{\mathbf{i}}\right\rangle\right]_{l}+\mu\left(\frac{\partial}{\partial x_{k}}\left[\mathbf{S}_{\mathbf{i}}\left\langle\mathbf{u}_{\mathbf{i}}\right\rangle\right]_{j}+\frac{\partial}{\partial x_{j}}\left[\mathbf{S}_{\mathbf{i}}\left\langle\mathbf{u}_{\mathbf{i}}\right\rangle\right]_{k}\right), j, k, l=1,2$,

where $\sigma_{j k}^{E}$ and $\sigma_{j k}^{S}$ are the stress fields induced by $\left\langle\mathbf{u}_{\mathbf{i}}\right\rangle$ and $\mathbf{S}_{\mathbf{i}}\left\langle\mathbf{u}_{\mathbf{i}}\right\rangle$, respectively, while $\lambda$ and $\mu$ are the Lamé constants of the fractured rock. 
Substituting eqs (5) and (6) into eq. (9) yields

$\sigma_{j k}^{S}=-\mu \int_{-a}^{a}\left[\Delta \mathbf{u}_{\mathbf{i}}\left(\zeta_{1}, p_{1}, p_{2}\right)\right]_{l} T_{j k l}\left(x_{1}, x_{2} \mid \zeta_{1}, 0\right) d \zeta_{1}, j, k, l=1,2$,

where the expressions for $T_{j k l}$ are shown in Appendix A.

For dry open fractures, the normal and shear stresses applied on the fracture surface vanish and hence

$\sigma_{12}^{E}+\sigma_{12}^{S}=0,-a<x_{1}<a, x_{2}=0$,

and

$\sigma_{22}^{E}+\sigma_{22}^{S}=0,-a<x_{1}<a, x_{2}=0$.

Substituting eqs (4), (8) and (10) into eqs (11) and (12) yields

$\int_{-a}^{a} D_{1}\left(\zeta_{1}\right) T_{121}\left(x_{1}, 0 \mid \zeta_{1}, 0\right) d \zeta_{1}-e^{i k_{p} x_{1} \sin \theta}=0,-a<x_{1}<a$,

and

$\int_{-a}^{a} D_{2}\left(\zeta_{1}\right) T_{222}\left(x_{1}, 0 \mid \zeta_{1}, 0\right) d \zeta_{1}-e^{i k_{p} x_{1} \sin \theta}=0,-a<x_{1}<a$,

where

$D_{1}\left(\zeta_{1}\right)=\frac{\left[\Delta \mathbf{u}_{\mathbf{i}}\left(\zeta_{1}, p_{1}, p_{2}\right)\right]_{1}}{2 i\left(k_{\mathrm{p}} \cos \theta+\kappa\right) \sin \theta A e^{i k_{\mathrm{p}} p_{1} \sin \theta+i\left(k_{\mathrm{p}} \cos \theta+\kappa\right) p_{2}}}$,

and

$D_{2}\left(\zeta_{1}\right)=\frac{\left[\Delta \mathbf{u}_{\mathbf{i}}\left(\zeta_{1}, p_{1}, p_{2}\right)\right]_{2}}{i k_{\mathrm{p}} A e^{i k_{\mathrm{p}} p_{1} \sin \theta+i\left(k_{\mathrm{p}} \cos \theta+\kappa\right) p_{2}}\left[\left(k_{\mathrm{s}}^{2} / k_{\mathrm{p}}^{2}-2\right) \sin ^{2} \theta+k_{\mathrm{s}}^{2} / k_{\mathrm{p}}^{2}\left(\cos \theta+\kappa / k_{\mathrm{p}}\right)^{2}\right]}$.

The normal and shear displacement discontinuities across the fracture can be obtained by solving integral eqs (13) and (14). The coefficient $\kappa$ can then be calculated from the displacement discontinuities. To do so, we can first obtain $\mathbf{S}_{\mathbf{A}}\left\langle\mathbf{u}_{\mathbf{i}}\right\rangle$ by rewriting $\mathbf{S}_{\mathbf{i}}\left\langle\mathbf{u}_{\mathbf{i}}\right\rangle$ using the global coordinate system. Then, by substituting $\mathbf{S}_{\mathbf{A}}\left\langle\mathbf{u}_{\mathbf{i}}\right\rangle$ and eqs (15) and (16) into eq. (3) and comparing the result with eq. (2), we can obtain the expression for $\kappa$ (Kawahara 1992):

$\kappa=v \phi_{1} \gamma k_{\mathrm{p}} \sin 2 \theta \sin \theta+v \phi_{2} \frac{k_{\mathrm{p}}}{2 \gamma \cos \theta}\left(1-2 \gamma \sin ^{2} \theta\right)^{2}$

where $\gamma=V_{\mathrm{s}}^{2} / V_{\mathrm{p}}^{2}$, and $\phi_{j}$ has the following form:

$\phi_{j}\left(k_{\mathrm{p}}, \theta\right)=\int_{-a}^{a} D_{j}\left(\zeta_{1}\right) e^{-i k_{\mathrm{p}} \zeta_{1} \sin \theta} d \zeta_{1}, j=1,2$.

After obtaining $\kappa$, the frequency dependency of the phase velocity $V_{\mathrm{pe}}$ and attenuation factor $Q_{\mathrm{p}}^{-1}$ of the $P$ wave can be calculated as (Kawahara \& Yamashita 1992):

$V_{\mathrm{pe}} / V_{\mathrm{p}}=1-\frac{\cos \theta}{k_{\mathrm{p}}} \operatorname{Re} \kappa$

and

$Q_{\mathrm{p}}^{-1}=2 \frac{\cos \theta}{k_{\mathrm{p}}} \operatorname{Im} \kappa$

\section{MODEL FOR THE FLUID SATURATED FRACTURE CASE}

When the fractures are saturated with a viscous fluid, the fracture displacement discontinuities will be influenced by the fluid. However, the relation between the scattered wavefield and the fracture displacement discontinuities remains unchanged and hence eqs (17)-(20) are still applicable (Kawahara 1992). Therefore, to obtain the $P$-wave scattering dispersion and attenuation for fluid filled fractures, the corresponding displacement discontinuities $\left(D_{1}\right.$ and $\left.D_{2}\right)$ across the fractures need to be calculated. In this case, the fluid will apply both normal and shear stresses on the fracture surface as follows:

$\sigma_{12}^{E}+\sigma_{12}^{S}=-i \omega \eta \frac{\left[\Delta \mathbf{u}_{\mathbf{i}}\left(x_{1}, p_{1}, p_{2}\right)\right]_{1}}{\beta},-a<x_{1}<a, x_{2}=0$,

$\sigma_{22}^{E}+\sigma_{22}^{S}=K_{f} \frac{\left[\Delta \mathbf{u}_{\mathbf{i}}\left(x_{1}, p_{1}, p_{2}\right)\right]_{2}}{\beta},-a<x_{1}<a, x_{2}=0$, 
where $\eta$ and $K_{\mathrm{f}}$ are the shear viscosity and bulk modulus of the fluid, respectively. The shear stress is caused by the viscous friction between the fluid and the fracture surface (Kawahara \& Yamashita 1992), whereas the normal stress is equal to the fluid pressure in the fracture, which is generated due to the fracture volume compression (or extension). It should be noted that, as we consider the fluid with relatively high viscosity, the fluid flow inside the fracture is ignored and hence the fluid pressure is not equilibrated, and can vary with the location $x_{1}$.

By substituting eqs (4), (8) and (10) into eqs (21) and (22), we obtain

$\int_{-a}^{a} D_{1}\left(\zeta_{1}\right) T_{121}\left(x_{1}, 0 \mid \zeta_{1}, 0\right) d \zeta_{1}-e^{i k_{\mathrm{p}} x_{1} \sin \theta}=\frac{i \omega \eta}{\mu} \frac{D_{1}\left(x_{1}\right)}{\beta},-a<x_{1}<a$,

and

$\int_{-a}^{a} D_{2}\left(\zeta_{1}\right) T_{222}\left(x_{1}, 0 \mid \zeta_{1}, 0\right) d \zeta_{1}-e^{i k_{\mathrm{p}} x_{1} \sin \theta}=-\frac{K_{f}}{\mu} \frac{D_{2}\left(x_{1}\right)}{\beta}-a<x_{1}<a$,

where the relationships between $D_{j}$ and $\left[\Delta \mathbf{u}_{\mathbf{i}}\right]_{j}$ are given by eqs (15) and (16).

Hence, by solving eqs (23) and (24), the values of $D_{1}$ and $D_{2}$ can be obtained. It is difficult to solve eqs (23) and (24) analytically. However, it is straightforward to solve them numerically using the method proposed by Kawahara \& Yamashita (1992). This can be done by first normalizing eqs (23) and (24) as follows:

$\int_{-1}^{1} \hat{D}_{1}\left(\hat{\zeta}_{1}\right) \hat{T}_{121}\left(s, 0 \mid \hat{\zeta}_{1}, 0\right) d \hat{\zeta}_{1}-e^{i \hat{k}_{\mathrm{p}} s \sin \theta}=\frac{i \omega \eta a}{\mu} \frac{\hat{D}_{1}(s)}{\beta},-1<s<1$,

and

$\int_{-1}^{1} \hat{D}_{2}\left(\hat{\zeta}_{1}\right) \hat{T}_{222}\left(s, 0 \mid \hat{\zeta}_{1}, 0\right) d \hat{\zeta}_{1}-e^{i \hat{k}_{\mathrm{p}} s \sin \theta}=-\frac{K_{f} a}{\mu} \frac{\hat{D}_{2}(s)}{\beta},-1<s<1$,

where $\hat{\zeta}_{1}, s, \hat{k}_{\mathrm{p}}, \hat{D}_{1}, \hat{D}_{2}, \hat{T}_{121}$, and $\hat{T}_{222}$ are normalized values by the half of the fracture length $a$ as follows:

$\hat{\zeta}_{1}=\zeta_{1} / a$,

$s=x_{1} / a$,

$\hat{k}_{\mathrm{p}}=a k_{\mathrm{p}}$,

$\hat{D}_{j}=D_{j} / a, j=1,2$

and

$\hat{T}_{j 2 j}=a^{2} T_{j 2 j}, j=1,2$.

Then, eqs (25) and (26) can be discretized as follows:

$\sum_{n=1}^{M-1}\left(T_{m n}^{121}-\delta_{m n} \frac{i \omega \eta a}{\mu \beta}\right) \hat{D}_{1 n}=e^{i \hat{k}_{\mathrm{p}} s_{m} \sin \theta}, \quad m=1, \ldots, M-1$

and

$\sum_{n=1}^{M-1}\left(T_{m n}^{222}+\delta_{m n} \frac{K_{f} a}{\mu \beta}\right) \hat{D}_{2 n}=e^{i \hat{k}_{\mathrm{p}} s_{m} \sin \theta}, \quad m=1, \ldots, M-1$

where $s_{m}=-1+m \Delta s$, and $\Delta s=2 / M, T_{m n}^{121}$ and $T_{m n}^{222}$ are calculated from $\hat{T}_{121}$ and $\hat{T}_{222}$ as follows:

$T_{m n}^{j 2 j}=\int_{s_{n}-\Delta s / 2}^{s_{n}+\Delta s / 2} \hat{T}_{j 2 j}\left(s_{m}, 0 \mid \hat{\zeta}_{1}, 0\right) d \hat{\zeta}_{1}, j=1,2$

The expressions of $T_{m n}^{121}$ and $T_{m n}^{222}$ are given in Appendix B. Hence, $\hat{D}_{1}$ and $\hat{D}_{2}$ can be computed by solving the matrix eqs (32) and (33) using the corresponding numerical algorithm. Then, $\phi_{1}$ and $\phi_{2}$ can be calculated as follows:

$\phi_{j}=a^{2} \hat{\phi}_{j}, j=1,2$,

where

$\hat{\phi}_{j}=\sum_{m=1}^{M-1} \hat{D}_{j m} e^{-i \hat{k}_{\mathrm{p}} s_{m} \sin \theta} \Delta s, j=1,2$. 
Substituting eq. (35) into eq. (17) yields

$\kappa=\varepsilon \hat{\phi}_{1} \gamma k_{\mathrm{p}} \sin 2 \theta \sin \theta+\varepsilon \hat{\phi}_{2} \frac{k_{\mathrm{p}}}{2 \gamma \cos \theta}\left(1-2 \gamma \sin ^{2} \theta\right)^{2}$

where $\varepsilon=v a^{2}$ is called the fracture density for the slit fractures (e.g. Kachanov \& Sevostianov 2005). It can be seen from eq. (37) that the expression for the fracture density is the same for the dry and saturated fractures, the saturating fluid only influences the normal and shear displacement discontinuities across the fractures. Furthermore, we can observe from eqs (32) and (33) that in the low-frequency limit, the normalized fracture displacement discontinuities for the saturated fractures are affected by the fracture aspect ratio, whereas those for the dry fractures are independent of the fracture aspect ratio. This is consistent with the Eshelby model (Mura 1987; Sevostianov \& Kachanov 1999).

After the value of $\kappa$ has been obtained, the $P$-wave scattering dispersion and attenuation for the fluid saturated fracture case can be obtained from eqs (19) and (20). It can be noted that the scattering dispersion and attenuation are not controlled by the number density $v$, but the combination of the number density and half of the fracture length (i.e. fracture density $\varepsilon$ ).

\section{NUMERICAL EXAMPLE}

\subsection{Parameters}

In this section, we give a numerical example to illustrate the $P$-wave scattering dispersion and attenuation in the fractured reservoir. We consider a carbonate reservoir with negligible background porosity (e.g. Rashid et al. 2015). The properties of the isotropic elastic background

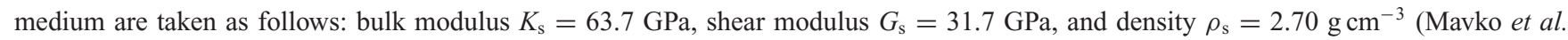
2009). Large 2-D fractures (fracture corridors) are developed in the reservoir with their planes parallel to each other and centres distributed randomly and sparsely in the background medium. It is assumed that the size of the fractures is identical to a length $2 a$ of $40 \mathrm{~m}$ and a thickness $\beta$ of $0.4 \mathrm{~m}$ (aspect ratio $\alpha=0.01$ ). The fractures are saturated with a viscous fluid, with a bulk modulus $K_{\mathrm{f}}$ of $2.25 \mathrm{GPa}$ (close to that of water) and a relatively high shear viscosity $\eta$ of $0.1 \mathrm{~Pa} * \mathrm{~s}$.

Using these parameters, we can first analyse the variations of the scattering dispersion and attenuation with frequency, fracture density, and incidence angle. Then, we can vary the fracture thickness and the saturating fluid properties to study their influence on the scattering dispersion and attenuation. Since the peak scattering attenuation occurs when the seismic wavelength is comparable to the fracture length (e.g. Kawahara \& Yamashita 1992), the frequencies studied here range from 10 to $1000 \mathrm{~Hz}$ due to the large fracture length (40 m).

\subsection{Variations of $P$-wave velocity and attenuation with frequency, fracture density, and incidence angles}

The variations of the $P$-wave velocity and attenuation with frequency $f$, fracture density $\varepsilon$, and incidence angle $\theta$ are shown in Figs 2 and 3 , respectively. It can be seen that in the low-frequency regime (Rayleigh scattering domain), the $P$-wave velocity decreases slightly with the frequency. Then it increases rapidly when the resonant (or Mie) scattering (e.g. Ishimaru 1978) occurs at around $100 \mathrm{~Hz}$, for which the incidence wavelength is of the order of size of the fracture length. In the high-frequency regime, the $P$-wave velocity tends to the value of the background medium with some small fluctuations. These small fluctuations are probably due to the interference pattern variations of the scattered wavefield generated by the fracture tips (Kawahara \& Yamashita 1992). This behaviour of the $P$-wave velocity with the frequency is similar to the case of dry fractures (Kawahara 1992). This is due to the assumptions in the model that the fluid is isolated in the fractures and no fluid flow occurs, hence, the effects of the fluid are similar to those of the elastic solid. For the dry fractures, the fracture infill material can be treated as an elastic solid with zero moduli. Therefore, the $P$-wave velocity for the fluid saturated fracture case behaves similarly with the dry fracture case, but with a smaller dispersion magnitude due to the reduced stiffness contrast between the fractures and the background medium. In terms of the effects of the fracture density, it can be found from eqs (19) and (37) that the $P$-wave velocity dispersion increases linearly with the fracture density due to the application of the Foldy approximation, which gives the solution in the first order in $\varepsilon$ (Keller 1964; Ishimaru 1978). Comparing the wave dispersion at different incidence angles (Figs 2a-c), we note that the magnitude of the dispersion decreases with the incidence angles to the smallest value at $90^{\circ}$. Note that while the dispersion at $90^{\circ}$ is very small, it is not zero. This is due to the fact that, when the $P$-wave propagates parallel to the fracture plane, the compaction and extension of the rock in this direction will also induce a small normal displacement discontinuity across the fractures due to the Poisson ratio effects. It can also be noted that, the characteristic frequency for the dispersion is found to shift slightly between the oblique incidence angles and the normal (or grazing) incidence angle $\left(\theta=0^{\circ}\right.$ or $\left.90^{\circ}\right)$. The reason is that the dispersion and attenuation at the normal (or grazing) incidence angle is only controlled by the normal fracture displacement discontinuity, whereas that at oblique incidence angles is controlled by both the normal and shear displacement discontinuities across the fracture.

The scattering attenuation (Fig. 3) first increases with the frequency and reaches the peak at around $100 \mathrm{~Hz}$ when the incidence wavelength is of similar size with the fracture length. This corresponds to the resonant (or Mie) scattering domain, where the $P$-wave velocity increases rapidly with the frequency. The attenuation (inverse quality factor $Q_{\mathrm{p}}^{-1}$ ) in the low- and high-frequency regimes is approximately proportional to $f^{2}$ and $f^{-1}$, respectively. This is also observed by Yamashita (1990) and Kawahara \& Yamashita (1992) for the dry fracture case. In the high-frequency regime, the attenuation decreases with some small fluctuations, which is caused by interference pattern variations of the scattered wavefields from the fracture tips (Kawahara \& Yamashita 1992). Due to the assumption of random and sparse distribution 


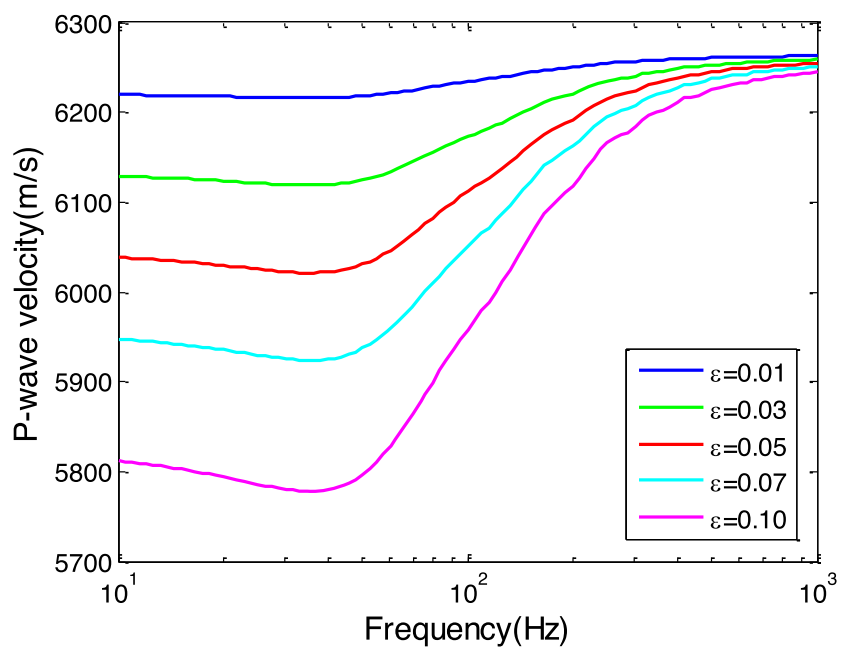

(a)

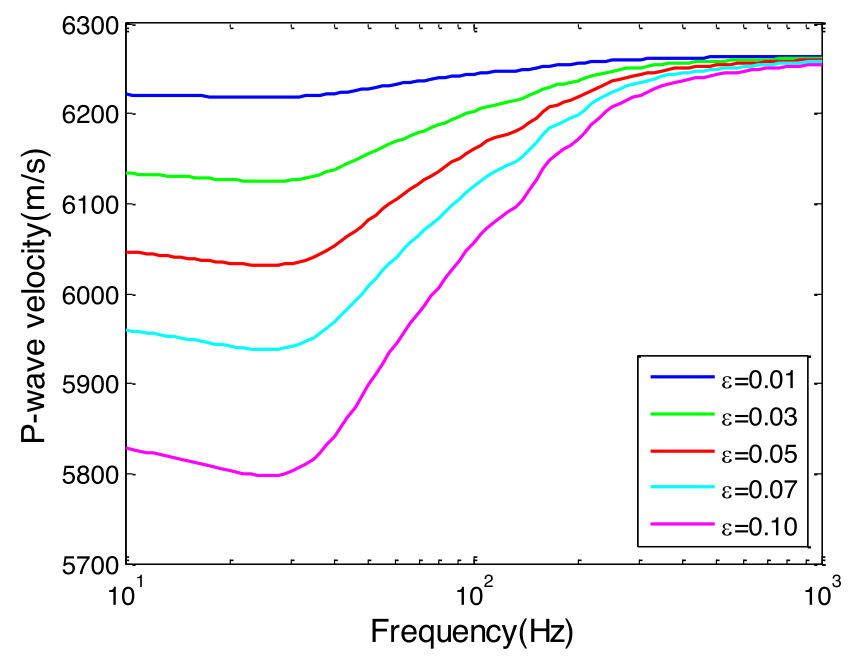

(b)

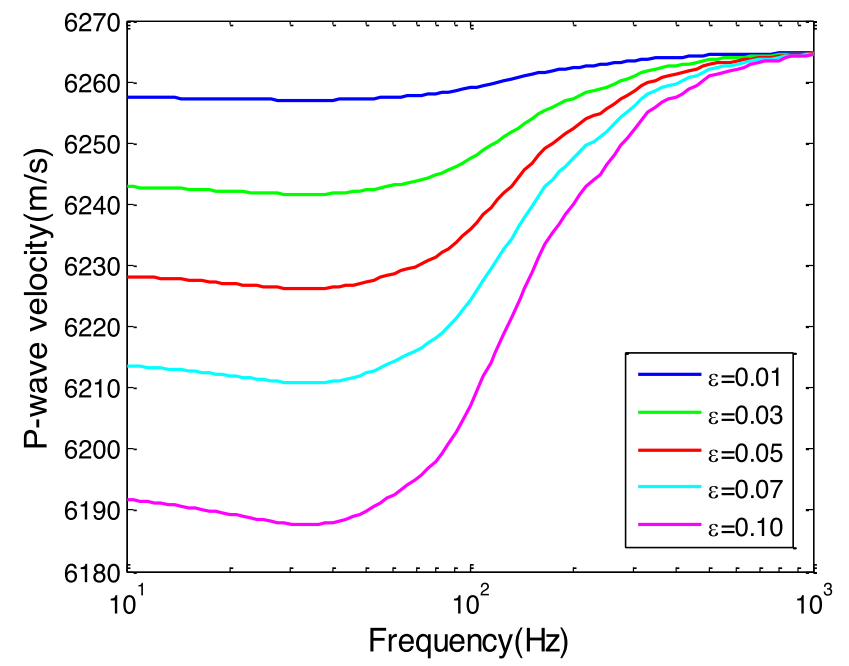

(c)

Figure 2. Variations of $P$-wave velocity with frequency $f$, fracture density $\varepsilon$, and incidence angle $\theta$. (a) $\theta=0^{\circ}$ (normal incidence); (b) $\theta=45^{\circ}$; (c) $\theta=90^{\circ}$ (grazing incidence). Note the much smaller scale of the $y$-axis for case (c) to show the small velocity dispersion. 


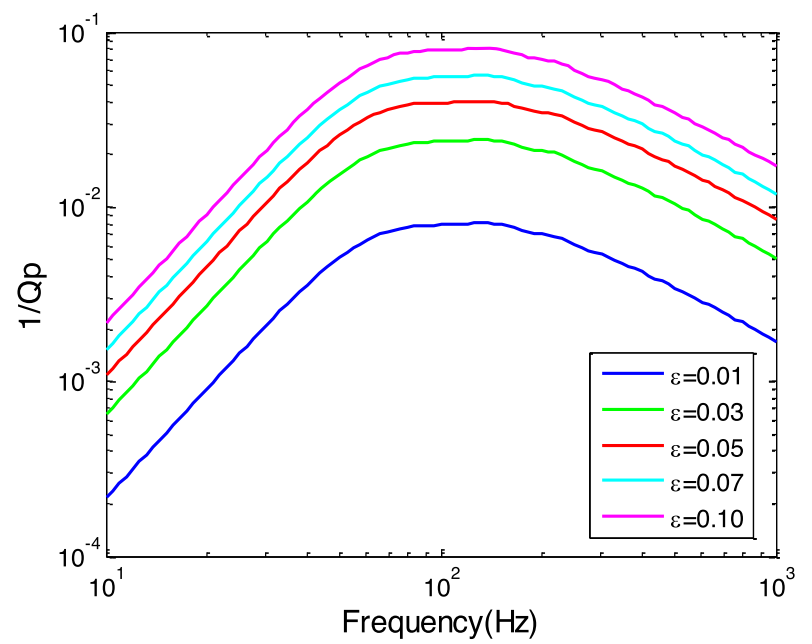

(a)

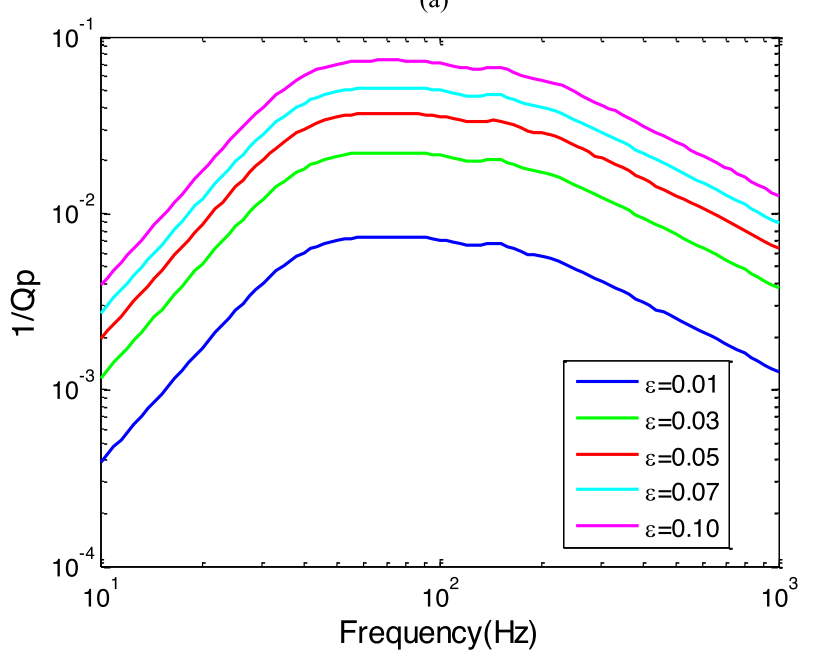

(b)

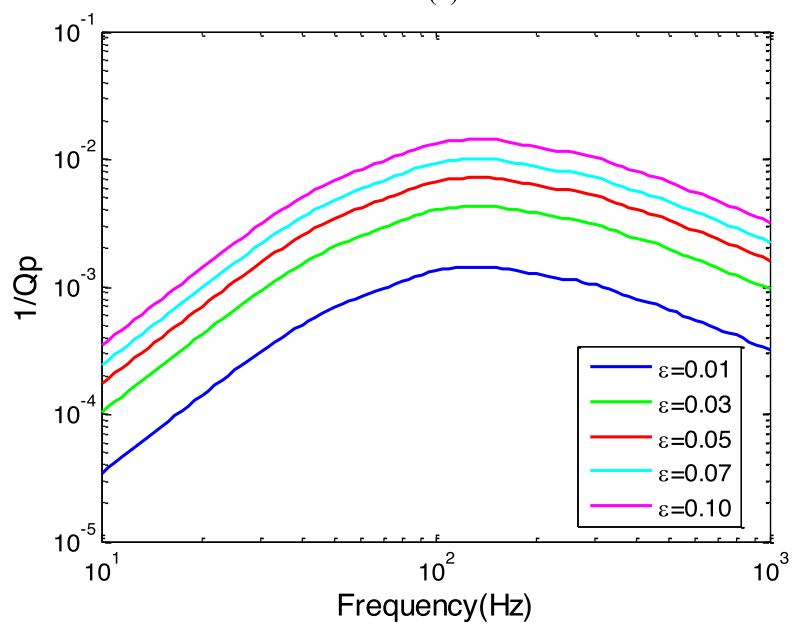

(c)

Figure 3. Variations of $P$-wave scattering attenuation with frequency $f$, fracture density $\varepsilon$, and incidence angle $\theta$. (a) $\theta=0^{\circ}$ (normal incidence); (b) $\theta=45^{\circ}$; (c) $\theta=90^{\circ}$ (grazing incidence). Note the different scale of the $y$-axis for case (c) to show the small attenuation. 


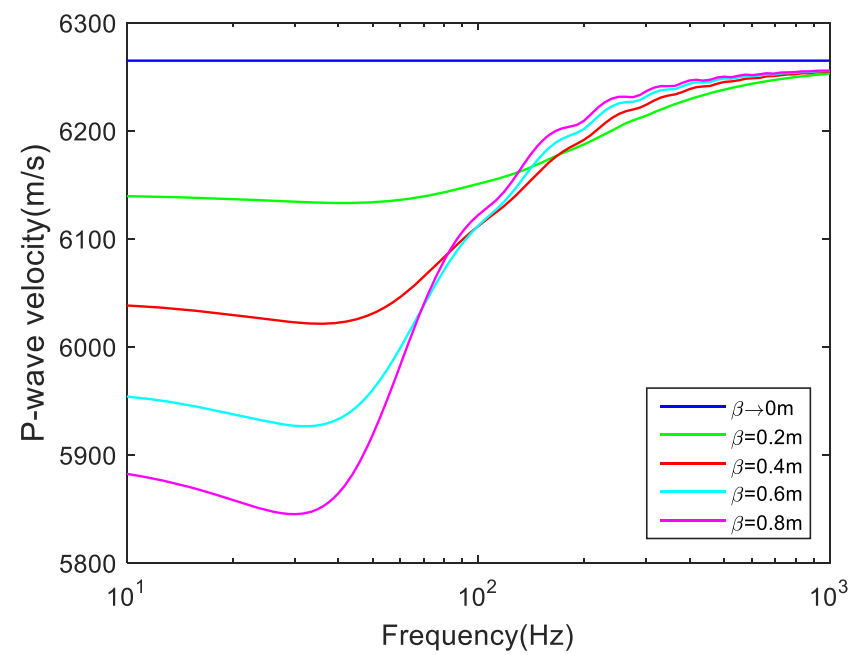

(a)

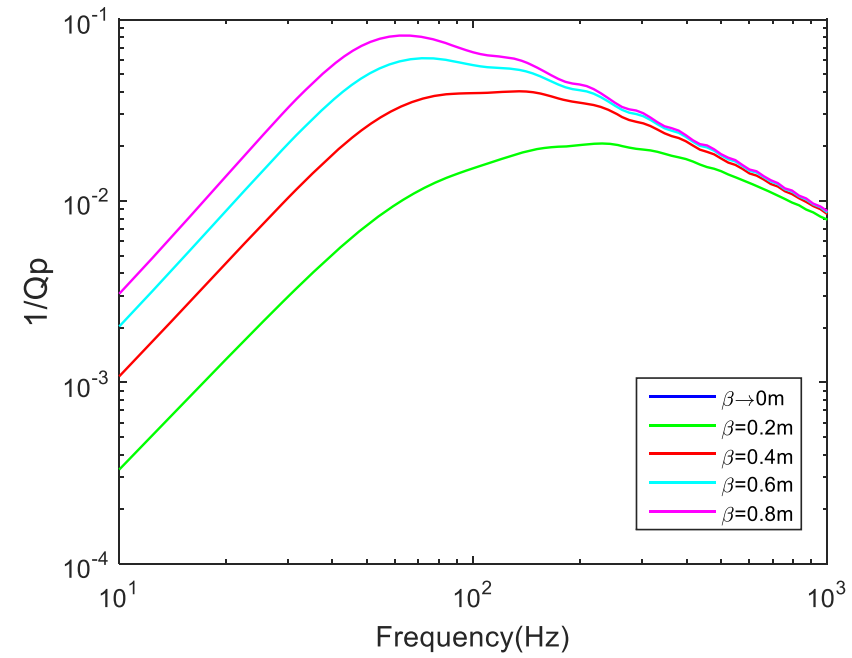

(b)

Figure 4. Effects of the fracture thickness on the $P$-wave scattering dispersion and attenuation at the normal incidence $\left(\theta=0^{\circ}\right)$ and different frequencies $f$ $(\varepsilon=0.05)$. (a) $P$-wave dispersion; (b) attenuation. Note that the dispersion and attenuation for the infinitesimal thickness case are zero.

of fractures, similar with the dispersion, the attenuation is also found to increase with the fracture density linearly. When changing the incidence angles from $0^{\circ}$ to $90^{\circ}$, the attenuation decreases and reaches the smallest (but non-zero) value at $90^{\circ}$. Similar to the dispersion, the characteristic frequency for the attenuation also shifts slightly between the normal (or grazing) incidence case and the oblique incidence case.

\subsection{Effects of fracture thickness on the scattering dispersion and attenuation}

The effects of fracture thickness on the scattering dispersion and attenuation are found to be similar at different incidence angles. Hence, we only show the results at the normal incidence $\left(\theta=0^{\circ}\right)$ in Fig. $4(\varepsilon=0.05)$. In the low-frequency regime, it is found that the fracture thickness has significant influence on the $P$-wave velocity. In agreement with the predictions by the static Eshelby model (Mura 1987; Sevostianov $\&$ Kachanov 1999), the $P$-wave velocity decreases with fracture thickness. However, in the high-frequency regime, it is interesting to note that the $P$-wave velocity increases with the fracture thickness. This is due to the fact that the magnitude of the scattering dispersion increases with the fracture thickness, as shown in Fig. 4 . Hence, the $P$-wave velocity in the Mie scattering regime increases more rapidly for the case with larger fracture thickness. This will thus result in the reversed trend of the $P$-wave velocity with the fracture thickness in the high-frequency regime.

It can be noted here that there is no dispersion or attenuation for the infinitesimal fracture thickness case at the normal incidence. This is because both the normal and shear displacement discontinuities across the fracture vanish in this case. According to eqs (17)-(20), the $P$-wave dispersion and attenuation will thus vanish. Similarly, at incidence angles other than $0^{\circ}$, the $P$-wave dispersion and attenuation also vanish if the fracture thickness is infinitesimal. Hence, the $P$-wave velocity equals to that of the background medium at all frequencies and remains unaffected by the incidence angle in this case. This is different from the predictions of the Eshelby model (Mura 1987; Sevostianov \& Kachanov 1999) in the low-frequency limit, which predicts the largest velocity at the incidence angle of $0^{\circ}$ or $90^{\circ}$, but smallest at $45^{\circ}$. The 


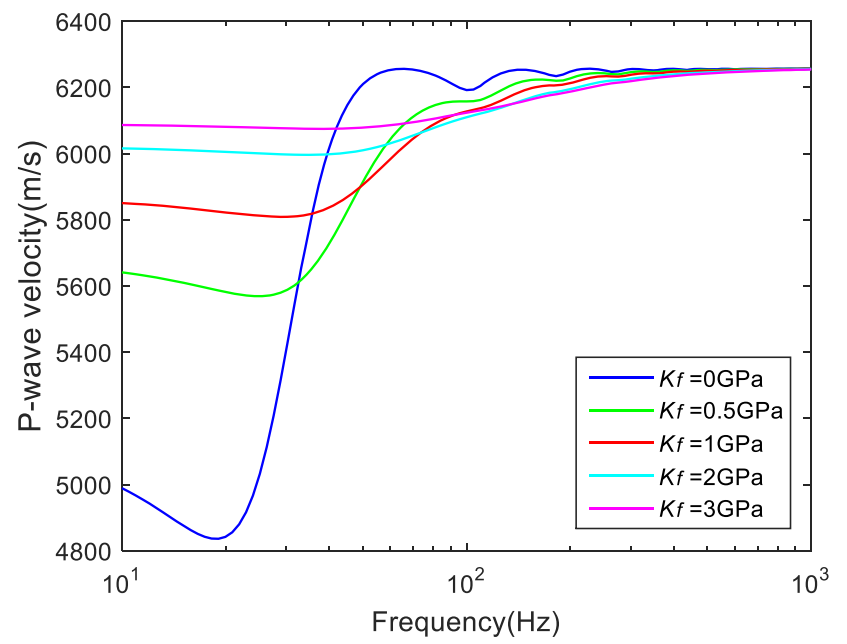

(a)

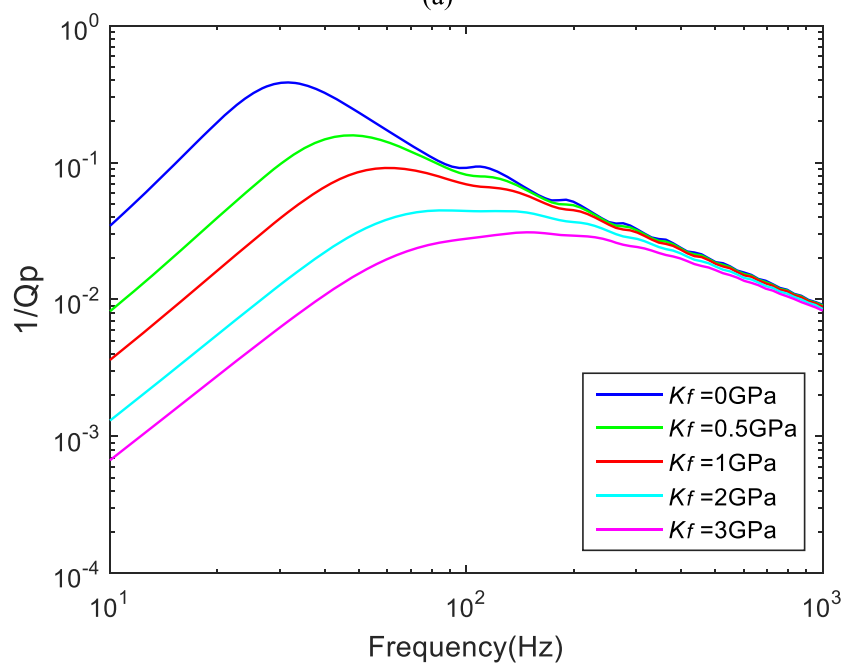

(b)

Figure 5. Effects of the fluid bulk modulus on the $P$-wave scattering dispersion and attenuation at the normal incidence $\left(\theta=0^{\circ}\right)$ and different frequencies $f$ $(\varepsilon=0.05)$. (a) $P$-wave dispersion; (b) attenuation.

reason is that while the normal fracture displacement discontinuity given by our model and the Eshelby model both vanish for the infinitesimal thickness case, the shear displacement discontinuity is different in these two models. In our model, we consider the viscous fluid and hence the viscous friction between the fracture surface and the fluid leads to zero shear fracture displacement discontinuity for the infinitesimal thickness case, as indicated by eq. (23). However, no viscous friction exists in Eshelby model and hence the shear fracture displacement discontinuity does not vanish at the incidence angles other than $0^{\circ}$ and $90^{\circ}$, which is largest at $45^{\circ}$. It should be noted that, while not shown here, the results of our model are consistent with those given by Eshelby model when the fluid viscosity reduces to zero.

We also find that the attenuation increases with the fracture thickness. This is primarily due to the increase of the normal displacement discontinuity across the fracture with the fracture thickness. The effects of fracture thickness on the attenuation are found to be significant in the low-frequency regime. However, in the high-frequency regime, the effects of the fracture thickness are smaller, they decrease with the frequency and vanish when the seismic wavelength is much smaller than the fracture size. Furthermore, the peak of the attenuation (characteristic frequency) shifts towards the low-frequency regime when the fracture thickness increases.

\subsection{Effects of the saturating fluid properties on the scattering dispersion and attenuation}

Similar to the effects of fracture thickness, the effects of the bulk modulus of the saturating fluid on the scattering dispersion and attenuation are similar at different incidence angles. Hence, we only show the results at the normal incidence $\left(\theta=0^{\circ}\right)$ in Fig. $5(\varepsilon=0.05)$. Significant influence of the fluid bulk modulus on the velocity dispersion is observed. The scattering dispersion decreases with the increase of the fluid bulk modulus and hence the largest dispersion occurs for the dry fracture case. The results for the dry fracture case in Fig. 5 are the same as those given by the model of Kawahara (1992). In the low-frequency regime, the $P$-wave velocity increases with the fluid bulk modulus, which agrees with the predictions of the static Eshelby model (Mura 1987; Sevostianov \& Kachanov 1999). However, in the high-frequency regime, 
the $P$-wave velocity decreases with the fluid bulk modulus. These different trends of the $P$-wave velocity with the fluid bulk modulus in the low- and high-frequency regimes were also observed by Sabina et al. (1993) and Smyshlyaev \& Willis (1993b). The reason is that the $P$-wave velocity increases more rapidly in the Mie scattering domain for the cases with lower fluid bulk modulus, which results in the reversal of the trends of the $P$-wave velocity with the fluid bulk modulus in the high-frequency regime.

The scattering attenuation decreases with the fluid bulk modulus. This effect is significant in the low-frequency regime, but negligible in the high-frequency regime. The characteristic frequency of the peak attenuation is found to shift towards the high-frequency regime with the increase of the fluid bulk modulus. Here, we observe that the effects of the fluid bulk modulus on the scattering dispersion and attenuation are opposite to those of the fracture thickness. This can be explained by eq. (24). From this equation, we can see that increasing the fluid bulk modulus has similar effects on the normal fracture displacement discontinuity as decreasing the fracture thickness.

Apart from the fluid bulk modulus, the fluid viscosity should also have some effects on the scattering dispersion and attenuation. This is expected because the viscous friction operating at the fracture surface affects the shear fracture displacement discontinuity and hence the scattering dispersion and attenuation. From the wave motion characteristics of the $P$-wave, it is expected that the shear displacement discontinuity should be largest at an incidence angle of around $45^{\circ}$ (Kawahara \& Yamashita 1992). Hence, the effects of fluid viscosity should be most obvious at this angle, which is shown in Fig. 6. However, contrary to our expectation, the curves for different fluid viscosities nearly overlap each other and hence the effect of the fluid viscosity is negligible, even when the viscosity reaches 10 Pa.s. The reason can be found in eq. (23), which shows the effects of fluid viscosity in the right-hand side. As the term $(\omega \eta) / \mu$ is usually much smaller than 1 , the shear displacement discontinuity across the fracture will be nearly unaffected by the fluid viscosity and hence its value will be close to the case with non-viscous fluid. Therefore, the fluid viscosity has little effects on the scattering dispersion and attenuation for the studied configurations. However, it should be noted that, when the fracture thickness is extremely small, the effects of fluid viscosity may be significant, as indicated by eq. (23). Furthermore, for the case with low fluid viscosity, the non-uniform normal displacement discontinuity across the fracture may induce the fluid flow inside the fractures. This will affect the dispersion and attenuation of the $P$ wave, which is not considered in our current model. Its effects will be discussed in the discussion section.

\section{COMPARISON WITH EXPERIMENTAL DATA}

\subsection{Experiment configuration and sample parameters}

To validate our theoretical model, we compare the theoretical predictions with the ultrasonic measurements on synthetic rock samples containing penny-shaped fractures performed by Wei et al. (2013). The host medium of the samples was constructed using a rock powderepoxy mixture by a layering technique. The resulting layered medium has equal thickness for each layer. The layered background medium shows very weak anisotropy (Wei et al. 2013) and hence can be treated as isotropic. The porosity of the background medium is negligible and the density is $1.66 \mathrm{~g} \mathrm{~cm}^{-3}$. Fractures are added by placing randomly, on the surface of each layer, penny-shaped inclusions. Thus, the fractures are aligned to each other and distributed randomly on the surface of each layer. The penny-shaped inclusions are punched out from a block sample made of a mixture of silica rubber and epoxy. The $P$-wave velocity of this mixture is measured giving an average value of $1350 \mathrm{~m} \mathrm{~s}{ }^{-1}$. However, the $S$ wave of this mixture cannot be identified due to its very small amplitude and signal-to-noise ratio (Fig. 7). This means that the silica rubber-epoxy mixture behaves similar to a fluid, that is, its shear modulus is either zero or much smaller than its bulk modulus. This indicates that for small deformations (such as in the ultrasonic measurements), the effect of the silica rubber-epoxy mixture properties on the overall elastic properties of the fractured rock is similar to that of a fluid. This conclusion, although somewhat counterintuitive, follows from the elastic/viscoelastic correspondence principle (Hashin 1970). According to this principle, effective properties of a two-phase viscoelastic composite can be computed using the same mixture theories as for elastic media by replacing elastic component properties with complex-valued moduli of viscoelastic components computed in the frequency domain. Since the shear modulus of the silica rubber-epoxy mixture is negligibly small, the effect of this mixture on the overall elastic properties of the fractured rock should be similar to that of a fluid based on the correspondence principle. Even though the shear modulus is probably real or nearly real for the silica rubber-epoxy mixture but purely imaginary for a Newtonian fluid, this difference is insignificant as long as both are much smaller than their respective bulk moduli, and the shear modulus of the host medium. Recently, this correspondence principle was used to compute the effect of squirt flow between cracks and pores from a solution of an equivalent elasticity problem (e.g. Glubokovskikh \& Gurevich 2017). Despite the fact that fluid flows but solid does not, this approach leads to exactly the same solution as obtained using linearized Navier-Stokes equations for the pore fill (Gurevich et al 2010). Hence, the theoretical model proposed in Section 3 can be used for the predictions of the experimental results here. Using the $P$-wave velocity measured above $\left(1350 \mathrm{~m} \mathrm{~s}^{-1}\right)$ and the density $\left(1.09 \mathrm{~g} \mathrm{~cm}^{-3}\right)$ of the silica rubber-epoxy mixture, its bulk modulus can be calculated to be around $2.02 \mathrm{GPa}$ while its shear modulus is negligibly small. The other details on the sample microstructure and the procedure of constructing the samples are given in Wei (2004) and Wei et al. (2013). Seven synthetic rock samples (Fig. 8) were then constructed: one reference sample (with no fractures) and six fractured samples with fixed fracture density (around 0.083) and radius (1.5 mm), but varying fracture thickness (Table 1).

Due to the small fracture size, the wave scattering can occur in the ultrasonic frequency range and hence we measure the $P$-wave velocity and attenuation using the ultrasonic pulse transmission method in the direction perpendicular and parallel to the fracture plane. The measurement system consists of the Panametrics-NDT 5077PR Pulser-Receiver connected to a TK-DPO3102 digital oscilloscope and a 


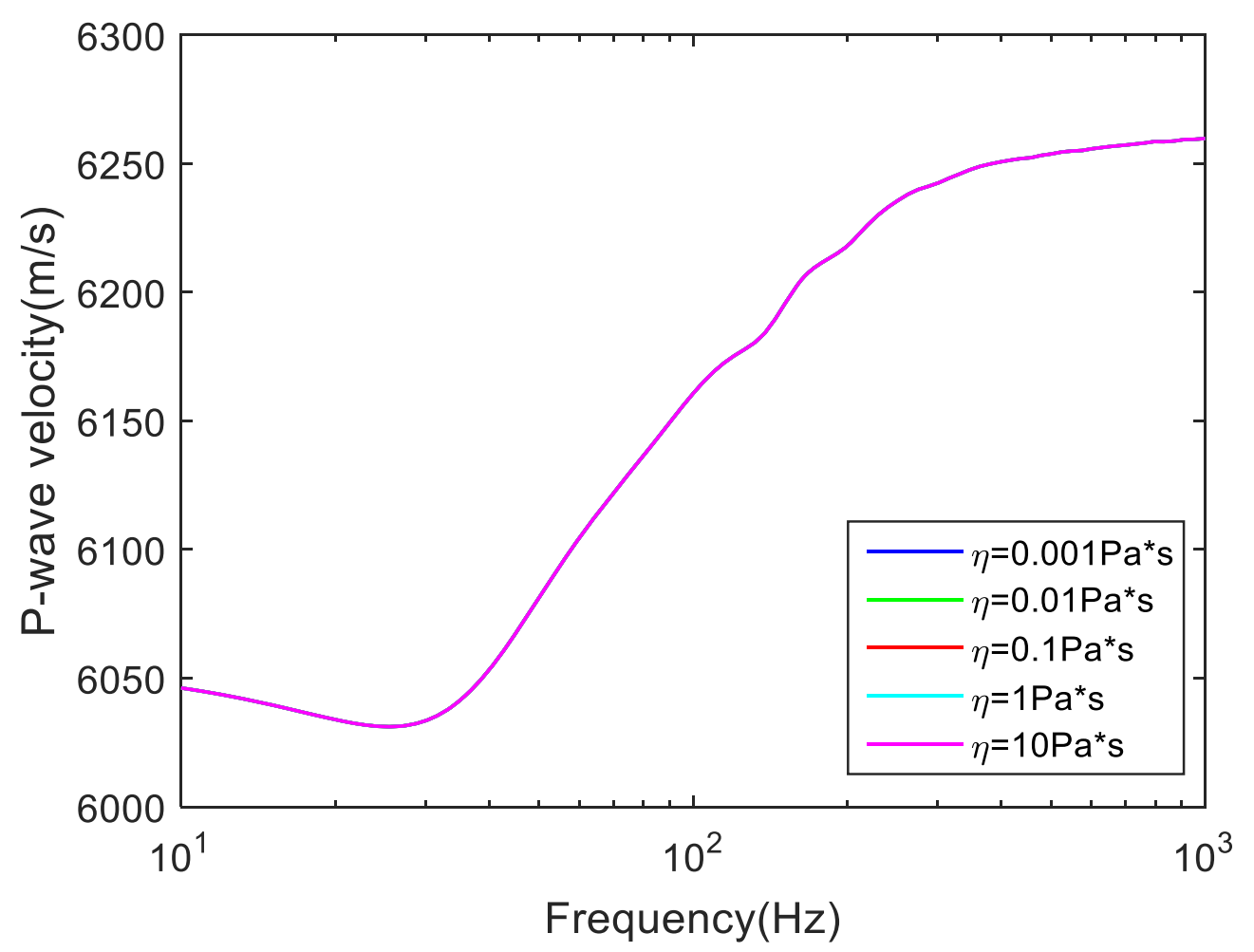

(a)

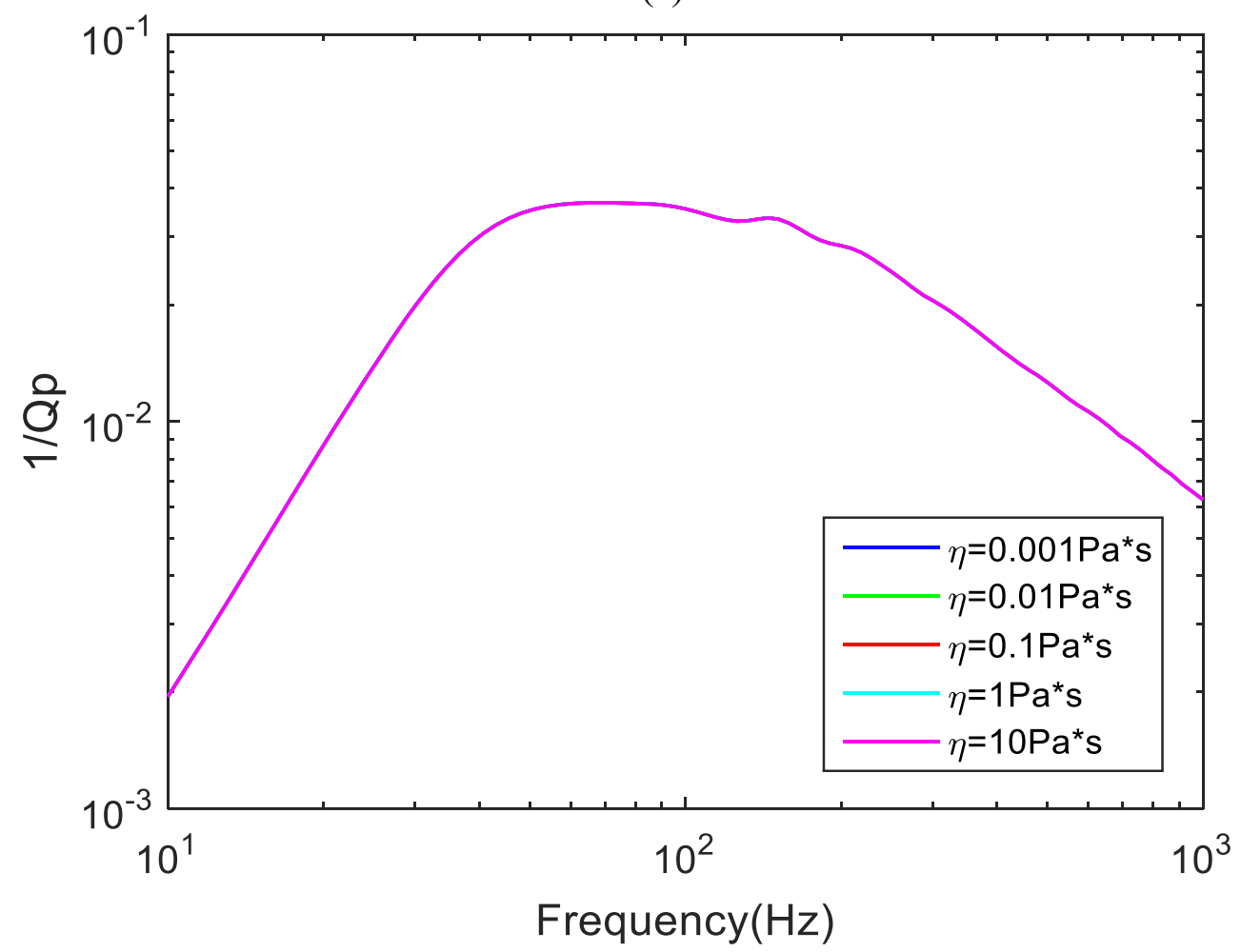

(b)

Figure 6. Effects of fluid viscosity on the $P$-wave scattering dispersion and attenuation under different frequencies $f$ at the incidence angle of $45^{\circ}$. (a) $P$-wave dispersion; (b) attenuation. 


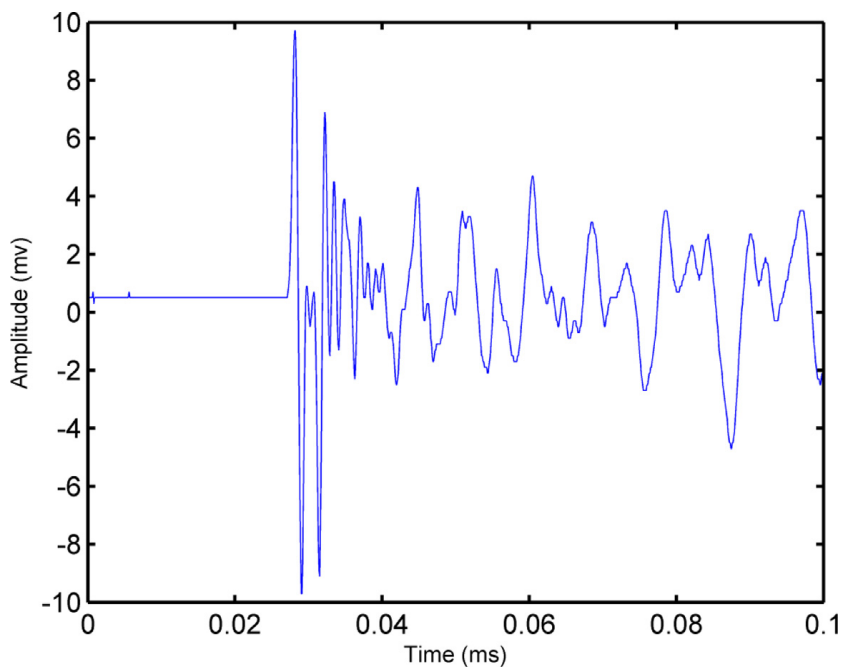

Figure 7. Measurement of $S$-wave velocity of the fracture infilling material (silica rubber-epoxy mixture). Note that the first arrival is $P$ wave, not $S$ wave. The $S$ wave cannot be identified due to its very small amplitude and signal-to-noise ratio.

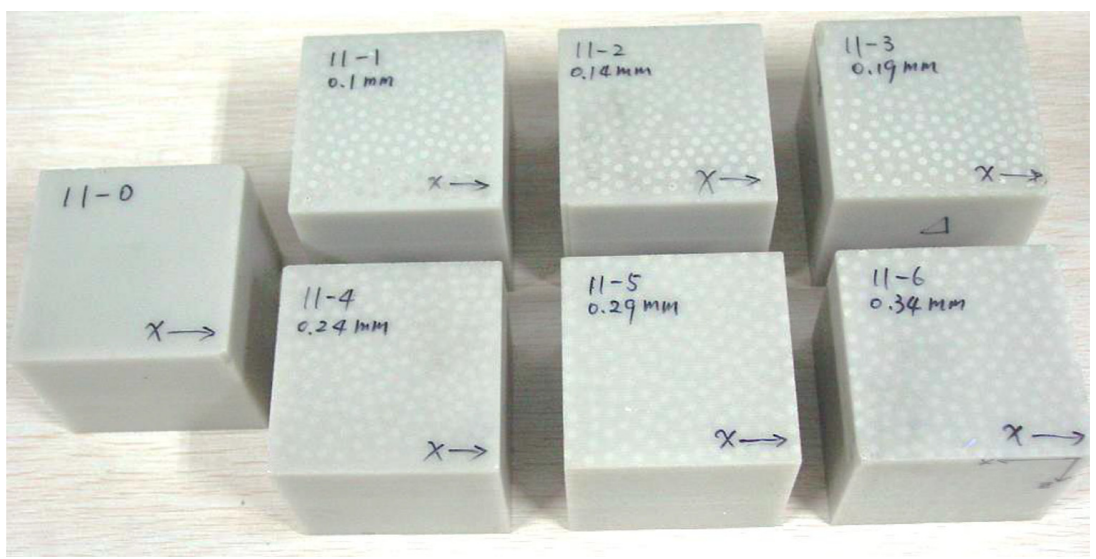

Figure 8. Synthetic rock samples containing penny-shaped fractures. The sample $11-0$ is the reference sample with no fractures, whereas samples $11-1$ to 11-6 have the same fracture density and radius, but with the increasing fracture thickness, as indicated in the figure $(0.1-0.34 \mathrm{~mm})$.

Table 1. Distribution of the fracture thickness of the samples.

\begin{tabular}{lccc}
\hline Sample no. & Fracture thickness $(\mathrm{mm})$ & Sample no. & Fracture thickness $(\mathrm{mm})$ \\
\hline $11-1$ & 0.10 & $11-4$ & 0.24 \\
$11-2$ & 0.14 & $11-5$ & 0.29 \\
$11-3$ & 0.19 & $11-6$ & 0.34 \\
\hline
\end{tabular}

Table 2. Physical parameters of transducers used to record $P$ - and $S$-wave seismograms.

\begin{tabular}{lccccc}
\hline & \multicolumn{2}{c}{$P$ wave } & & \multicolumn{2}{c}{$S$ wave } \\
\cline { 2 - 3 } \cline { 5 - 6 } $\begin{array}{l}\text { Nominal frequency } \\
(\mathrm{MHz})\end{array}$ & Catalogue number & $\begin{array}{c}\text { Transducer diameter } \\
(\mathrm{mm})\end{array}$ & & Catalogue number & $\begin{array}{c}\text { Transducer diameter } \\
(\mathrm{mm})\end{array}$ \\
\hline 1 & $\mathrm{~V} 102-\mathrm{RB}$ & 25 & & V152-RB & 25 \\
0.25 & $\mathrm{~V} 1012$ & 39 & & V150-RB & 25 \\
0.1 & V1011 & 39 & & V1548 & 25 \\
\hline
\end{tabular}

PC. To obtain the results at different frequencies, three pairs of transducers are used with the nominal frequencies of $1,0.25$ and $0.1 \mathrm{MHz}$, respectively. The physical parameters of the transducers are shown in Table 2. More information about the transducers can be accessed at the website of the manufacturer (www.olympus-ims.com/en/ultrasonic-transducers). By applying a Fourier transform to the recorded waveforms of $P$ waves transmitted through the reference sample, we can obtain their centroid frequencies, which are close to those of the incident $P$ waves. Hence, we can obtain the centroid frequencies of the incident $P$ waves for the three pairs of transducers, whose values are around 0.66, 0.21 and $0.097 \mathrm{MHz}$, respectively. At different centroid frequencies, the $P$ - and $S$-wave velocities of the background medium (reference 
Table 3. Elastic moduli of the background medium at different centroid frequencies of the incident $P$ waves.

\begin{tabular}{lcc}
\hline Centroid frequency $(\mathrm{MHz})$ & Bulk modulus $(\mathrm{GPa})$ & Shear modulus $(\mathrm{GPa})$ \\
\hline 0.66 & 9.39 & 3.81 \\
0.21 & 9.15 & 3.76 \\
0.097 & 9.30 & 3.61 \\
\hline
\end{tabular}

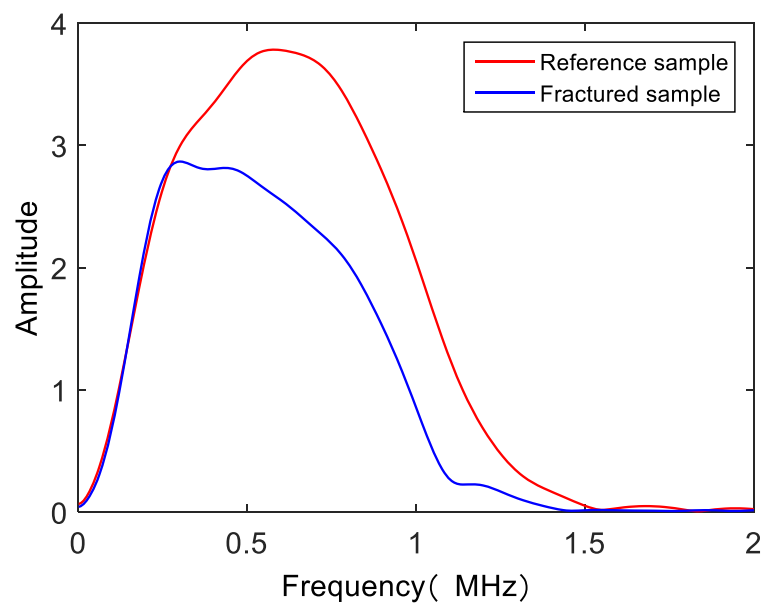

Figure 9. An example of the amplitude spectra of the transmitted $P$ waves through reference and fractured samples, respectively.

sample) are measured. Combining with the density of the background medium $\left(1.66 \mathrm{~g} \mathrm{~cm}^{-3}\right.$, the elastic moduli of the background medium are obtained which vary slightly with the frequency, as shown in Table 3. This indicates that the background medium has slightly viscoelastic (or near-elastic) properties. Hence, apart from the $P$-wave scattering, a small intrinsic attenuation may also occur in the background medium. As the host medium of the fractured samples has nearly the same properties as the reference sample, the intrinsic attenuation should be similar between them. Hence, the intrinsic attenuation in the background can be excluded by comparing the results with the measurements on the reference sample, using the following expressions (e.g. Tang et al. 1990):

$\ln \frac{\left|S_{2}(f)\right|}{\left|S_{1}(f)\right|}+\ln \frac{\left|G_{1}(f)\right|}{\left|G_{2}(f)\right|}=\frac{\pi L_{1} f}{V_{\mathrm{p}}} \frac{1}{Q_{\text {int }}}-\frac{\pi L_{2} f}{V_{\mathrm{pe}}}\left(\frac{1}{Q_{\text {int }}}+\frac{1}{Q_{\text {sca }}}\right)$,

and

$G_{i}(f)=\frac{\gamma}{i k_{\mathrm{p}}}\left[1-\left(1-\frac{\xi_{i}^{2}}{2 k_{\mathrm{p}}^{2} r^{2}}\right)\left[J_{0}\left(\xi_{i}\right)+i J_{1}\left(\xi_{i}\right)\right] e^{-i \xi_{i}}-\frac{\xi_{i}^{2}}{k_{\mathrm{p}}^{2} r^{2}}\left(\frac{i J_{1}\left(\xi_{i}\right)}{\xi_{i}}\right) e^{-i \xi_{i}}\right], \quad i=1,2$,

$\xi_{i}=\frac{k_{p}}{2}\left(\sqrt{4 r^{2}+L_{i}^{2}}-L_{i}\right), \quad i=1,2$

where $1 / Q_{\text {int }}$ and $1 / Q_{\text {sca }}$ are intrinsic and scattering attenuation, respectively; $f$ is the measurement frequency; $S_{1}$ and $S_{2}$ are the amplitude spectra of the transmitted $P$ waves through the reference and fractured samples, respectively; $L_{1}$ and $L_{2}$ are the length of the reference and fractured samples in the wave propagation direction, respectively; $r$ is the radius of the transducer; $J_{0}$ and $J_{1}$ are Bessel functions of the first kind with order zero and one, respectively. Note that the second term on the left-hand side of eq. (38) is the correction of the geometric spreading effects (Tang et al. 1990).

In this experiment, the size for the reference and fractured samples is similar $(\sim 68 \times 68 \times 62 \mathrm{~mm})$. Hence, the intrinsic attenuation $1 / Q_{\text {int }}$ in eq. (38) is cancelled and the following approximation is obtained:

$\ln \frac{\left|S_{2}(f)\right|}{\left|S_{1}(f)\right|}=-\frac{\pi L_{2} f}{V_{\mathrm{pe}}} \frac{1}{Q_{\text {sca }}}$.

Thus, we can estimate the $P$-wave scattering attenuation using eq. (41) through the spectral ratio method (e.g. Bath 1974; Ganley \& Kanasewich 1980; Mavko et al. 2009). The full measured data for the velocities and waveforms are given in Wei et al. (2013). An example of the amplitude spectra $S_{1}$ and $S_{2}$ is given in Fig. 9.

The results for the velocities and estimated scattering attenuation for the fractured samples are shown in Figs 10 and 11 . We can observe notable $P$-wave dispersion and attenuation. To validate our theoretical model, we can compute dispersion and attenuation theoretically, and compare the theoretical predictions with the experimental results. When carrying out the theoretical predictions, it should be noted that our model is for 2-D slit fractures while experiments use 3-D penny-shaped fractures. Since the wave scattering is controlled by the fracture density for both the 2-D and 3-D cases (e.g. Zhang \& Gross 1993a,b), we use the same fracture density in the 2-D model as that for the 3-D samples for the comparison of the theoretical predictions with the experimental results. Furthermore, as the primary component of the 


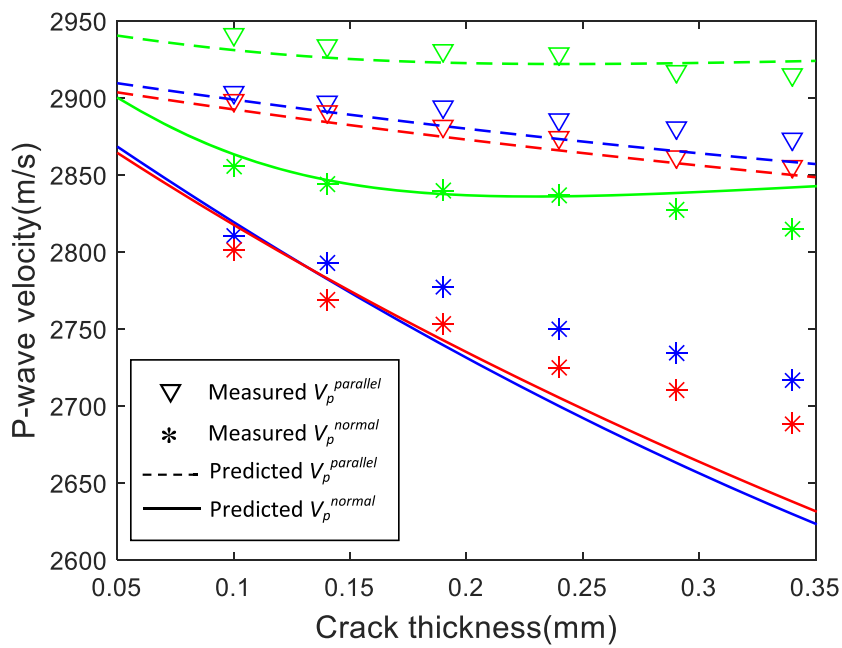

Figure 10. Measured and predicted $P$-wave velocities of the samples in the directions parallel and perpendicular to the fracture plane under different fracture thicknesses and centroid frequencies. The green, blue and red colours represent the velocities measured at the centroid frequencies of $0.66,0.21$ and $0.097 \mathrm{MHz}$, respectively. The dashed and solid lines represent the theoretical predictions in the directions parallel and perpendicular to the fracture plane, respectively. The triangles and the stars are the corresponding experimental measurement results.

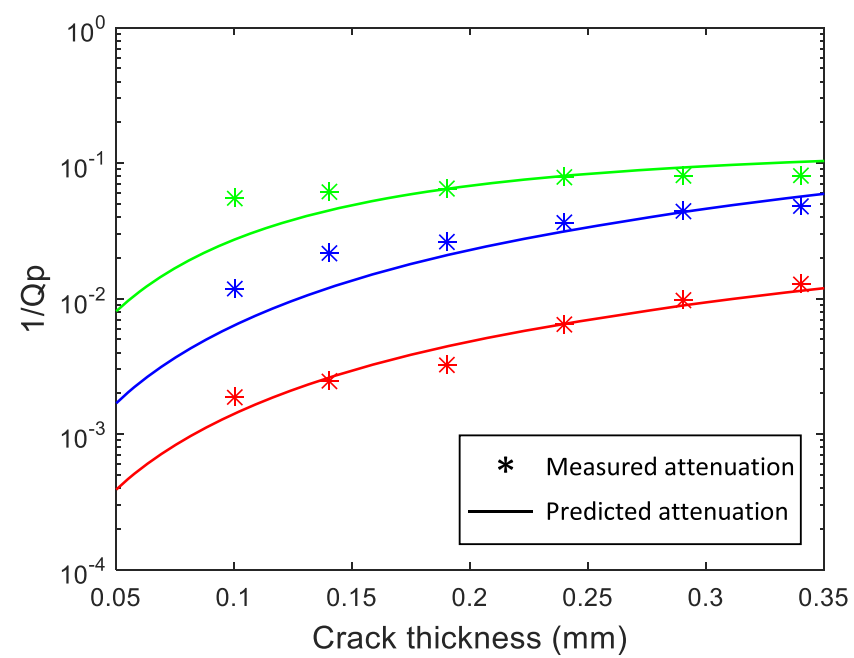

(a)

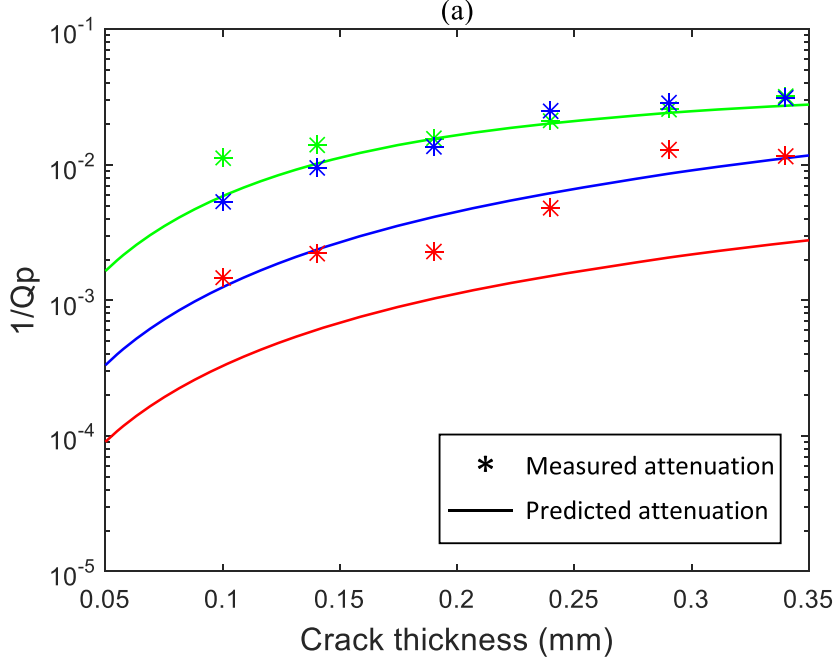

(b)

Figure 11. Measured and predicted $P$-wave attenuation in the directions perpendicular (a) and parallel (b) to the fracture plane under different fracture thicknesses and frequencies. The green, blue and red colours represent the attenuation measured at the centroid frequencies of $0.66,0.21 \mathrm{and} 0.097 \mathrm{MHz}$, respectively. The solid lines and the stars are the theoretical predictions and the experimental measurement results, respectively. 
incident $P$ waves is that at the centroid frequencies, we use the centroid frequencies (instead of the nominal frequencies) as the inputs in the theoretical predictions. In addition, to account for the effects of the slightly viscoelastic properties of the background medium on the $P$-wave velocities, we use frequency-dependent elastic properties of the background medium (Table 3 ) in the theoretical predictions. Finally, the viscosity of the fluid-like mixture in the fractures is not measured as there is no shear displacement discontinuity across the fractures for the wave propagating perpendicular and parallel to the fracture plane. Hence, it is not needed in the theoretical predictions.

\subsection{Comparison of experimental results with theoretical predictions}

Fig. 10 shows the $P$-wave velocity measured at different frequencies and fracture thickness and the corresponding theoretical predictions. For the $P$-wave propagating parallel to the fracture plane, it can be seen that the measured and predicted velocities are in good agreement with each other. They both decrease with the fracture thickness and increase with the frequency. At higher frequency $(0.66 \mathrm{MHz})$, the velocities are obviously larger than those at lower frequencies $(0.097$ and $0.21 \mathrm{MHz})$, which should be due to the Mie scattering effects. At lower frequencies, if the elastic properties of the background medium are frequency-independent, the velocities given by the theoretical predictions will decrease slightly with the frequency due to the Rayleigh scattering effects. However, as the $P$-wave modulus of the background medium increases slightly with the frequency in this regime (Table 3), the trend of the velocities with the frequency is reversed.

When the wave propagates perpendicular to the fracture plane, the theoretical predictions are also in overall good agreement with the experimental results. Both of them show an increase of the velocity dispersion with the fracture thickness, which is consistent with the observations in the numerical example section. The primary discrepancy is the slightly different trends of the $P$-wave velocity with the frequency in the relatively low-frequency regime $(0.097$ and $0.21 \mathrm{MHz})$. In the theoretical predictions, the dispersion caused by Rayleigh scattering for samples with relatively low fracture thickness is small and hence the velocities increase slightly due to the small increase of the $P$-wave modulus of the background medium with the frequency. However, for samples with relatively large fracture thickness, the dispersion due to Rayleigh scattering becomes larger, the theoretical predictions thus give lower velocities at $0.21 \mathrm{MHz}$ than those at $0.097 \mathrm{MHz}$. Different from the theoretical predictions, the experimental results show an increase of $P$-wave velocities with the frequency for all the samples measured. The possible reason for the discrepancy is that our theoretical model is based on the Foldy approximation, which neglects interactions between the fractures. This holds when the fracture density is low and the fractures are distributed randomly in the samples. However, the fracture density of the samples $(0.083)$ is close to 0.1 and hence is not that low (e.g. Grechka \& Kachanov, 2006; Suzuki et al. 2013). Furthermore, while the fractures are distributed randomly on the surface of each layer of the background medium, they are not randomly distributed throughout the samples. Hence, the concentration of the relatively large number of fractures on the same plane may increase interaction between the fractures (especially for the cases with relatively large fracture thickness), which may change the trend of the $P$-wave velocity with the frequency. In addition, it should also be noted that our theoretical model deals with the 2-D slit fractures, whereas the fractures in the samples are 3-D (penny-shaped). The difference in the geometries of the fractures may also lead to discrepancies between the theoretical predictions and the experimental results.

The measured $P$-wave attenuation in the directions perpendicular and parallel to the fracture plane is shown in Fig. 11, along with the corresponding theoretical predictions. It can be seen that the theoretical predictions are in overall good agreement with the measured results. Both the theoretical predictions and the measured results show that the attenuation increases with the fracture thickness at different measurement frequencies, which is consistent with the observations in the numerical example. Moreover, the effects of the fracture thickness on the attenuation at higher frequencies $(0.66 \mathrm{MHz})$ are smaller than those at lower frequencies $(0.21$ and $0.097 \mathrm{MHz})$. Again, this is also observed in the numerical example. The discrepancies between the theoretical predictions and experimental results primarily occur in the direction parallel to the fracture plane at lower frequencies $(0.21$ and $0.097 \mathrm{MHz})$. The theoretical predictions give lower values of attenuation than the experimental results. The discrepancies may be due to the transmission loss occurred on the surface of the sample and also the increased relative error in the measurement of the small attenuation, which is amplified by the logarithmic scale.

\section{DISCUSSION}

In this paper, we have considered fractures filled with the fluid with a relatively high viscosity and hence the fluid flow inside the fractures should be negligible in the scattering frequency regime. However, if the fluid has low viscosity, due to the non-uniform normal displacement discontinuity across the fractures, the fluid flow can be induced inside the fractures in the scattering frequency regime. The fluid flow will induce the intrinsic attenuation and also affect the scattering attenuation, which are not considered in our current model. To study this effect, we can consider the limiting case when the fluid pressure is uniform in the fractures. This can be done by modifying the boundary condition (24) for the normal stress as follows:

$$
\int_{-a}^{a} D_{2}\left(\zeta_{1}\right) T_{222}\left(x_{1}, 0 \mid \zeta_{1}, 0\right) \mathrm{d} \zeta_{1}-e^{i k_{\mathrm{p}} x_{1} \sin \theta}=-\frac{K_{f}}{2 a \beta \mu} \int_{-a}^{a} D_{2}\left(\zeta_{1}\right) \mathrm{d} \zeta_{1} .
$$

Hence, using boundary conditions (23) and (42), the normal and tangential fracture displacement discontinuities can be computed by employing the same numerical method as before. Then, the $P$-wave dispersion and attenuation for this case can be calculated. The uniform fluid pressure case represents the situation where the fluid in the fractures is relaxed, whereas the no fluid flow case studied in Section 3 denotes the situation with unrelaxed fluid in the fractures. There is no intrinsic attenuation due to the fluid flow inside the fractures for both 


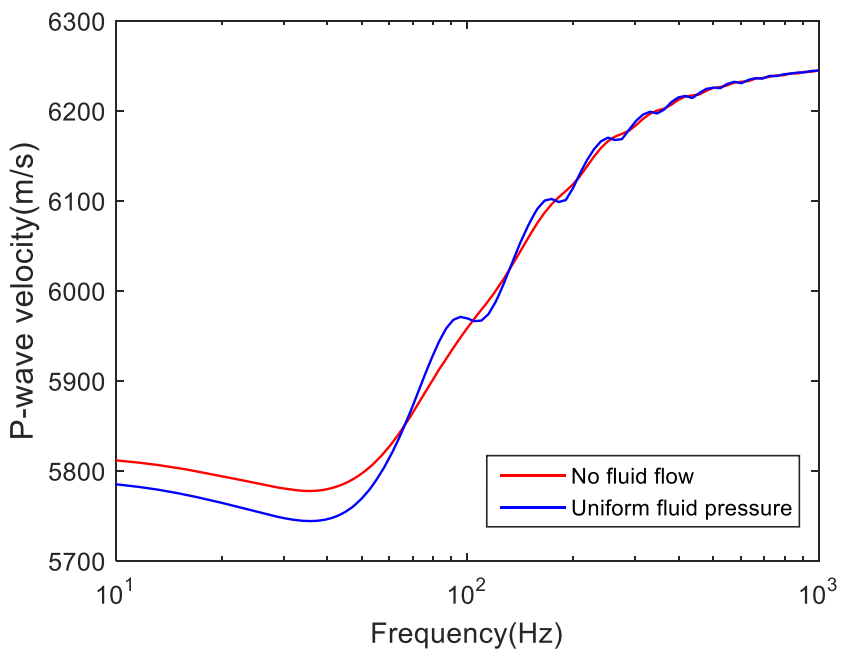

(a)

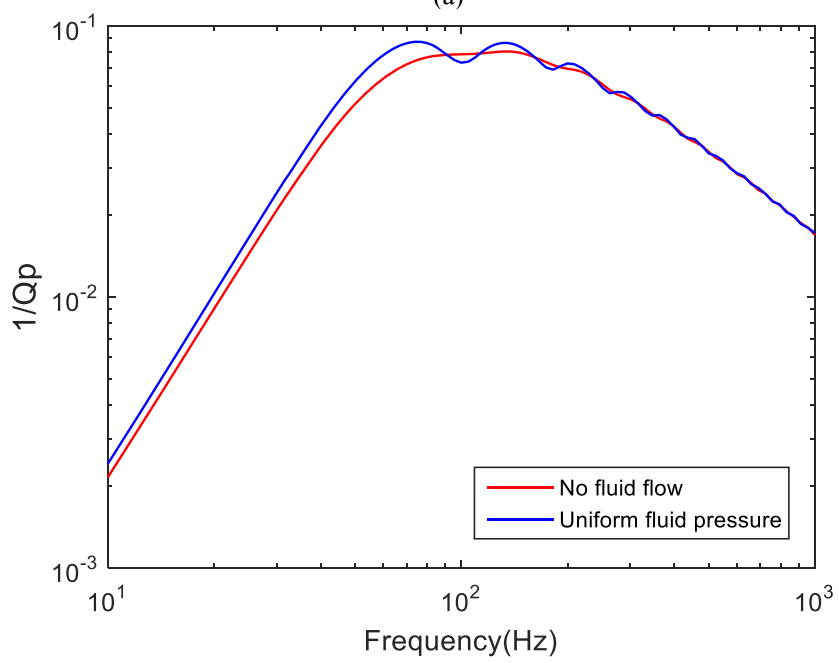

(b)

Figure 12. Comparison of the $P$-wave dispersion and attenuation between the no fluid flow case and the uniform fluid pressure case at the normal incidence $\left(\theta=0^{\circ}\right)$. (a) $P$-wave dispersion; (b) attenuation.

limiting cases. However, the differences in the velocities and attenuation at low frequencies (quasi-static regime) between these two cases can indicate the magnitude of the intrinsic attenuation. Furthermore, they can also show the largest possible effects of the fluid flow inside the fractures on the scattering dispersion and attenuation.

Fig. 12 shows the comparison between these two cases at the normal incidence $\left(\theta=0^{\circ}\right)$. The parameters used are the same with those in the numerical example section. The fracture density is assumed to be 0.1 . Since the normal fracture displacement discontinuity is largest at the normal incidence, the differences between these two limiting cases should also be largest at this incidence angle. We find that the differences between these two cases are small at low frequencies, where the no fluid flow case has higher velocity and lower attenuation than the uniform fluid pressure case. However, the differences are small even for a relatively large fracture density (0.1). The largest difference between the velocities does not exceed $35 \mathrm{~m} \mathrm{~s}^{-1}$ and for the attenuation is negligible. This indicates that the fluid flow inside the fractures induces negligible intrinsic attenuation and has very small effects on the scattering attenuation for the case with small aspect ratio ( 0.01$)$. Tests for larger aspect ratios (not presented here) show similar results even for the case with an aspect ratio of 0.1 . Hence, we can conclude that the fluid flow inside the fractures has negligible effects on the seismic dispersion and attenuation for the studied fracture configuration.

In addition, in our current model, we assume that fractures are embedded in an elastic non-porous background medium. However, for the saturated porous rock with aligned fractures, apart from the wave scattering effects and the fluid flow inside the fractures, the wave-induced fluid flow between the fractures and porous background medium can also occur, which will result in additional dispersion and attenuation of the seismic wave (e.g. Müller et al. 2010; Ba et al. 2011, 2014, 2017; Rubino \& Holliger 2013; Rubino et al. 2013). Hence, it is also essential to study this effect for such rock. To this end, Galvin \& Gurevich (2007) studied the scattering of a normal incident $P$ wave on a penny-shaped fracture in a fluid saturated porous medium. Both the elastic wave scattering and fluid flow effects are formulated in their approach. However, the scattering effects were then neglected by assuming the incompressible fluid in the fractures. Hence, by taking into account the compressibility of the fluid in the fracture and using a similar approach as that of Galvin \& Gurevich (2007), we can study both 
the effects of the wave scattering and the fluid flow between the fractures and the porous background medium. This work will be carried out in the future.

\section{CONCLUSIONS}

In this work, we study the $P$-wave dispersion and attenuation due to the scattering by the 2-D fluid saturated aligned fractures with finite thickness, which are embedded in an isotropic elastic solid. We assumed that the fractures are distributed randomly and sparsely, which allowed us to use the Foldy approximation, which, together with the representation theorem, gives the $P$-wave scattering dispersion and attenuation for the given displacement discontinuities across the fractures. Using the boundary conditions, the fracture displacement discontinuities can be computed numerically, which enables the calculation of $P$-wave dispersion and attenuation.

The behaviour of the theoretical results is illustrated using a numerical example. The analysis of this example shows that fracture thickness has significant influence on the $P$-wave dispersion and attenuation, especially in the low-frequency regime. In this regime, the $P$-wave velocity decreases with the increasing fracture thickness. However, due to the increase of the dispersion and attenuation with the fracture thickness, the trend of the $P$-wave velocity with the fracture thickness is reversed in the high-frequency regime. The fluid bulk modulus is also found to have significant effects on the dispersion and attenuation, but these effects are opposite to those of the fracture thickness. With respect to the fluid viscosity, its effects on the dispersion and attenuation are found to be negligible for the studied configurations.

To validate our 2-D theoretical model, we compare the theoretical predictions with the ultrasonic measurements on the 3-D fractured samples. The results show overall good agreement between the theoretical predictions and the experimental results. This work reveals the important effects of the fracture thickness and the properties of the saturating fluid on the $P$-wave scattering dispersion and attenuation. Hence, it provides a potential to extract these parameters from the seismic data.

\section{ACKNOWLEDGEMENTS}

JG and BG are grateful to the sponsors of the Curtin Reservoir Geophysics Consortium (CRGC) for financial support. JG acknowledges the financial support from National Key R\&D Program of China (No. 2018YFC0310105). DS, JW and PD acknowledge the financial support from National Natural Science Foundation of China (No. 41474112). The authors also thank Jun Kawahara for the kind assistance with the theory, and Stanislav Glubokovskikh and Vadim Lisitsa for useful discussion. The authors also greatly appreciate the insightful comments from the editor and two reviewers, which helped to improve the quality of this paper greatly.

\section{REFER EN CES}

Achenbach, J.D., 1973. Wave Propagation in Elastic Solids, Elsevier Science Publishers BV.

Ba, J., Carcione, J.M. \& Nie, J.X., 2011. Biot-Rayleigh theory of wave propagation in double-porosity media, J. geophys. Res., 116, B06202-1B06202-12.

Ba, J., Xu, W., Fu, L.-Y., Carcione, J.M. \& Zhang, L., 2017. Rock anelasticity due to patchy saturation and fabric heterogeneity: a double doubleporosity model of wave propagation, J. geophys. Res., 122, 1949-1976.

Ba, J., Zhang, L., Sun, W. \& Hao, Z., 2014. Velocity field of wave-induced local fluid flow in double-porosity media, Sci. China Phys. Mech. Astron., 57, 1020-1030.

Bath, M., 1974. Spectral Analysis in Geophysics, Elsevier Scientific Publishing Company.

Burns, D.R., Willis, M.E., Toksöz, M.N. \& Vetri, L., 2007. Fracture properties from seismic scattering, Leading Edge, 26, 1186-1196.

Bush, I., 2010. An integrated approach to fracture characterisation, Oil Rev. Middle East, 2, 88-91.

Caleap, M., Aristégui, C. \& Angel, Y.C., 2009. Effect of crack opening and orientation on dispersion and attenuation of antiplane coherent wave, Geophys. J. Int., 177, 1151-1165.

Eriksson, A.S., Bostrom, A. \& Datta, S.K., 1995. Ultrasonic wave propagation through a cracked solid, Wave Motion, 22, 297-310.

Foldy, L.L., 1945. The multiple scattering of waves I. General theory of isotropic scattering by randomly distributed scatterers, Phys. Rev., 67(3\&4), 107-119.

Galvin, R.J. \& Gurevich, B., 2007. Scattering of a longitudinal wave by a circular crack in a fluid-saturated porous medium, Int. J. Solids Struct., 44, 7389-7398.

Ganley, D.C. \& Kanasewich, E.R., 1980. Measurement of absorption and dispersion from check shot surveys, J. geophys. Res., 85(B10), 52195226.
Glubokovskikh, S. \& Gurevich, B., 2017. Optimal bounds for attenuation of elastic waves in porous fluid-saturated media, J. acoust. Soc. Am., 142, 3321-3329.

Grechka, V. \& Kachanov, M., 2006. Effective elasticity of rocks with closely spaced and intersecting cracks, Geophysics, 71(3), D85-D91.

Guo, J., Rubino, J.G., Barbosa, N.D., Glubokovskikh, S. \& Gurevich, B., 2018a. Seismic dispersion and attenuation in saturated porous rocks with aligned fractures of finite thickness: Theory and numerical simulations - Part 1: P-wave perpendicular to the fracture plane, Geophysics, 83(1), WA49-WA62.

Guo, J., Rubino, J.G., Barbosa, N.D., lubokovskikh, S. \& Gurevich, B., $2018 \mathrm{~b}$. Seismic dispersion and attenuation in saturated porous rocks with aligned fractures of finite thickness: Theory and numerical simulations -Part 2: Frequency-dependent anisotropy, Geophysics, 83(1), WA63WA71.

Gurevich, B., Makarynska, D., De Paula, O.B. \& Pervukhina, M., 2010. A simple model for squirt-flow dispersion and attenuation in fluid-saturated granular rocks, Geophysics, 75, N109-N120.

Gurevich, B., Zyrianov, V.B. \& Lopatnikov, S.L., 1997. Seismic attenuation in finely layered porous rocks: effects of fluid flow and scattering, Geophysics, 62(1), 319-324.

Hashin, Z., 1970. Complex moduli of viscoelastic composites - I. General theory and application to particulate composites, Int. J. Solids Struct., 6, 539-552.

Ishimaru, A., 1978. Wave Propagation and Scattering in Random Media, Academic Press.

Kachanov, M. \& Sevostianov, I., 2005. On quantitative characterization of microstructures and effective properties, Int. J. Solids Struct., 42, 309336.

Kawahara, J., 1992. Scattering of $P, S V$ waves by random distributions of aligned open cracks, J. Phys. Earth, 40, 517-524. 
Kawahara, J. \& Yamashita, T., 1992. Scattering of elastic waves by a fracture zone containing randomly distributed cracks, Pure appl. Geophys., 139, 121-144.

Keller, J.B., 1964. Stochastic equations and wave propagation in random media, Proc. Sympos. Appl. Math., 16, 145-170.

Keogh, P.S., 1986. High-frequency scattering of a normally incident plane compressional wave by a penny-shaped crack, Q. J. Mech. appl. Math., 39(4), 535-566.

Kikuchi, M., 1981. Dispersion and attenuation of elastic waves due to multiple scattering from inclusions, Phys. Earth planet. Inter, 25, $159-162$.

Krenk, S. \& Schmidt, H., 1982. Elastic wave scattering by a circular crack, Phil. Trans. R. Soc. Lond., A., 308, 167-198.

Kuster, G.T. \& Toksöz, M.N., 1974. Velocity and attenuation of seismic waves in two-phase media: Part I. Theoretical formulations, Geophysics, 39, 587-606.

Mal, A.K., 1970a. Interaction of elastic waves with a penny-shaped crack, Int. J. Eng. Sci., 8, 381-388.

Mal, A.K., 1970b. Interaction of elastic waves with a Griffith crack, Int. J. Eng. Sci., 8, 763-776.

Martin, P.A., 1981. Diffraction of elastic wave by a penny-shaped crack, Proc. R. Soc. A, 378, 263-285.

Martin, P.A. \& Rizzo, F.J., 1989. On boundary integral equations for crack problems, Proc. R. Soc. A, 421, 341-355.

Mavko, G., Mukerji, T. \& Dvorkin, J., 2009. The Rock Physics Handbook: Tools for Seismic Analysis of Porous Media, Cambridge Univ. Press.

Murai, Y., 2007. Scattering attenuation, dispersion and reflection of $S H$ waves in two-dimensional elastic media with densely distributed cracks, Geophys. J. Int., 168, 211-223.

Murai, Y., Kawahara, J. \& Yamashita, T., 1995. Multiple scattering of $S H$ waves in 2-D elastic media with distributed cracks, Geophys. J. Int., 122, 925-937.

Mura, T., 1987. Micromechanics of Defects in Solids, Martinus Nijhoff Publishers.

Müller, T.M., Gurevich, B. \& Lebedev, M., 2010. Seismic wave attenuation and dispersion resulting from wave-induced flow in porous rocks - A review, Geophysics, 75(5), 75A147-75A164.

Rashid, F., Glover, P.W.J., Lorinczi, P., Collier, R. \& Lawrence, J., 2015. Porosity and permeability of tight carbonate reservoir rocks in the north of Iraq, J. Petrol. Sci. Eng., 133, 147-161.

Rubino, J.G., Guarracino, L., Müller, T.M. \& Holliger, K., 2013. Do seismic waves sense fracture connectivity?, Geophys. Res. Lett., 40, 692-696.

Rubino, J.G. \& Holliger, K., 2013. Research note: seismic attenuation due to wave-induced fluid flow at microscopic and mesoscopic scales, Geophys. Prospect., 61, 882-889.

Sabina, F.J., Smyshlyaev, V.P. \& Willis, J.R., 1993. Self-consistent analysis of waves in a matrix-inclusion composite- I. Aligned spheroidal inclusions, J. Mech. Phys. Solids, 41, 1573-1588.

Sato, H., Fehler, M.C. \& Maeda, T., 2011. Seismic Wave Propagation and Scattering in the Heterogeneous Earth, 2nd edn, Springer.
Schoenberg, M. \& Douma, J., 1988. Elastic wave propagation in media with parallel fractures and aligned cracks, Geophys. Prospect., 36, 571-590.

Sevostianov, I. \& Kachanov, M., 1999. Compliance tensors of ellipsoidal inclusions, Int. J. Fract., 96, L3-L7.

Smyshlyaev, V.P. \& Willis, J.R., 1993a. Self-consistent analysis of waves in a matrix-inclusion composite-II. Randomly oriented spheroidal inclusions, J. Mech. Phys. Solids, 41, 1589-1598.

Smyshlyaev, V.P. \& Willis, J.R., 1993b. Self-consistent analysis of waves in a matrix-inclusion composite-III. A matrix containing cracks, J. Mech. Phys. Solids, 41, 1809-1824.

Suzuki, Y., Kawahara, J., Okamoto, T. \& Miyashita, K., 2006. Simulations of $S H$ wave scattering due to cracks by the 2-D finite difference method, Earth Planets Space, 58, 555-567.

Suzuki, Y., Shiina, T., Kawahara, J., Okamoto, T. \& Miyashita, K., 2013. Simulations of $P-S V$ wave scattering due to cracks by the 2-D finite difference method, Earth Planets Space, 65, 1425-1439.

Tang, X.M., Toksöz, M.N. \& Cheng, C.H., 1990. Elastic wave radiation and diffraction of a piston source, J. acoust. Soc. Am., 87(5), 1894-1902.

Timoshenko, S.P. \& Goodier, J.N., 1934. Theory of Elasticity, McGraw-Hill.

Vasconcelos, I. \& Jenner, E., 2005. Estimation of azimuthally varying attenuation from wide-azimuth $P$-wave data, in SEG Technical Program Expanded Abstracts, SEG, Tulsa, pp. 123-126.

Vlastos, S., Liu, E., Main, I.G. \& Li, X.-Y., 2003. Numerical simulation of wave propagation in media with discrete distributions of fractures: effects of fracture sizes and spatial distributions, Geophys. J. Int., 152, 649-668.

Vlastos, S., Liu, E., Main, I.G. \& Narteau, C., 2007. Numerical simulation of wave propagation in 2-D fractured media: scattering attenuation at different stages of the growth of a fracture population, Geophys. J. Int., 171, 865-880.

Vlastos, S., Liu, E., Main, I.G., Schoenberg, M., Narteau, C., Li, X.Y. \& Maillot, B., 2006. Dual simulations of fluid flow and seismic wave propagation in a fractured network: effects of pore pressure on seismic signature, Geophys. J. Int., 166, 825-838.

Wei, J., 2004. A physical model study of different crack densities, J. Geophys. Eng., 1, 70-76.

Wei, J., Di, B. \& Ding, P., 2013. Effect of crack aperture on $P$-wave velocity and dispersion, Appl. Geophys., 10(2), 125-133.

Wu, R. \& Aki, K., 1985. Scattering characteristics of elastic waves by an elastic heterogeneity, Geophysics, 50(4), 582-595.

Yamashita, T., 1990. Attenuation and dispersion of $S H$ waves due to scattering by randomly distributed cracks, Pure appl. Geophys., 132, 545-568.

Zhang, C.H. \& Achenbach, J. D., 1991. Effective wave velocity and attenuation in a material with distributed penny-shaped cracks, Int. J. Solids Struct., 27, 751-767.

Zhang, C.H. \& Gross, D., 1993a. Wave attenuation and dispersion in randomly cracked solids - I. Slit cracks, Int. J. Eng. Sci., 31, 841-858.

Zhang, C.H. \& Gross, D., 1993b. Wave attenuation and dispersion in randomly cracked solids - II. Penny-shaped cracks, Int. J. Eng. Sci., 31, 859-872.

\section{APPENDIX A: EXPRESSIONS OF $T_{j k l}$}

Expressions of $T_{j k l}$ are given by Kawahara \& Yamashita (1992), which are shown as follows:

$$
\begin{aligned}
T_{j k l}\left(x_{1}, x_{2} \mid \zeta_{1}, \zeta_{2}\right)= & T_{j k l}^{*}\left(x_{1}, x_{2} \mid \zeta_{1}, \zeta_{2}\right)+\frac{i}{4}\left[\left(\delta_{j l} \frac{\partial^{2}}{\partial x_{k} \partial x_{2}}+\delta_{k 2} \frac{\partial^{2}}{\partial x_{j} \partial x_{l}}+\delta_{k l} \frac{\partial^{2}}{\partial x_{j} \partial x_{2}}+\delta_{j 2} \frac{\partial^{2}}{\partial x_{k} \partial x_{l}}\right)\right. \\
& \left.\times H_{0}^{(1)}\left(k_{\mathrm{s}} R\right)-\frac{4}{k_{\mathrm{s}}^{2}} \frac{\partial^{4}}{\partial x_{j} \partial x_{k} \partial x_{l} \partial x_{2}}\left(H_{0}^{(1)}\left(k_{\mathrm{p}} R\right)-H_{0}^{(1)}\left(k_{\mathrm{s}} R\right)\right)\right],
\end{aligned}
$$

where $R^{2}=\left(x_{1}-\zeta_{1}\right)^{2}+\left(x_{2}-\zeta_{2}\right)^{2}$, and

$T_{111}^{*}\left(x_{1}, x_{2} \mid \zeta_{1}, \zeta_{2}\right)=\frac{i}{2}\left(1-2 \frac{k_{\mathrm{p}}^{2}}{k_{\mathrm{s}}^{2}}\right) \frac{\partial^{2}}{\partial x_{1} \partial x_{2}} H_{0}^{(1)}\left(k_{\mathrm{p}} R\right)$,

$T_{122}^{*}\left(x_{1}, x_{2} \mid \zeta_{1}, \zeta_{2}\right)=T_{212}^{*}\left(x_{1}, x_{2} \mid \zeta_{1}, \zeta_{2}\right)=T_{221}^{*}\left(x_{1}, x_{2} \mid \zeta_{1}, \zeta_{2}\right)=T_{111}^{*}\left(x_{1}, x_{2} \mid \zeta_{1}, \zeta_{2}\right)$, 
$T_{112}^{*}\left(x_{1}, x_{2} \mid \zeta_{1}, \zeta_{2}\right)=\frac{i}{4} \frac{k_{\mathrm{s}}^{2}}{k_{\mathrm{p}}^{2}}\left(1-2 \frac{k_{\mathrm{p}}^{2}}{k_{\mathrm{s}}^{2}}\right)\left(\frac{\partial^{2}}{\partial x_{1}^{2}}+\frac{\partial^{2}}{\partial x_{2}^{2}}\right) H_{0}^{(1)}\left(k_{\mathrm{p}} R\right)$,

$T_{121}^{*}\left(x_{1}, x_{2} \mid \zeta_{1}, \zeta_{2}\right)=T_{211}^{*}\left(x_{1}, x_{2} \mid \zeta_{1}, \zeta_{2}\right)=0$,

$T_{222}^{*}\left(x_{1}, x_{2} \mid \zeta_{1}, \zeta_{2}\right)=\frac{i}{4} \frac{k_{\mathrm{s}}^{2}}{k_{\mathrm{p}}^{2}}\left[\left(1-2 \frac{k_{\mathrm{p}}^{2}}{k_{\mathrm{s}}^{2}}\right)^{2} \frac{\partial^{2}}{\partial x_{1}^{2}}+\left(1-4 \frac{k_{\mathrm{p}}^{4}}{k_{\mathrm{s}}^{4}}\right) \frac{\partial^{2}}{\partial x_{2}^{2}}\right] H_{0}^{(1)}\left(k_{\mathrm{p}} R\right)$.

\section{APPENDIX B: NUMERICAL CALCULATION OF $T_{m n}^{j k l}$}

The expressions of $T_{m n}^{j k l}$ can also be found in Kawahara \& Yamashita (1992). They are repeated here for the convenience of the readers as follows:

When $m=n$,

$$
\begin{aligned}
T_{n n}^{121}= & -\frac{\hat{k}_{\mathrm{p}}^{2}-\hat{k}_{\mathrm{s}}^{2}}{\hat{k}_{\mathrm{s}}^{2}} \frac{4}{\pi \Delta s}-\frac{\hat{k}_{\mathrm{p}}^{4}+\hat{k}_{\mathrm{s}}^{4}}{\hat{k}_{\mathrm{s}}^{2}} \frac{i \Delta s}{8}+\frac{\Delta s}{4 \pi \hat{k}_{\mathrm{s}}^{2}}\left[\hat{k}_{\mathrm{p}}^{4} \log \frac{\hat{k}_{\mathrm{p}} \Delta s}{4}+\hat{k}_{\mathrm{s}}^{4} \log \frac{\hat{k}_{\mathrm{s}} \Delta s}{4}+\hat{k}_{\mathrm{p}}^{4}\left(C-\frac{3}{4}\right)+\hat{k}_{\mathrm{s}}^{4}\left(C-\frac{5}{4}\right)\right], \\
T_{n n}^{222}= & -\frac{\hat{k}_{\mathrm{p}}^{2}-\hat{k}_{\mathrm{s}}^{2}}{\hat{k}_{\mathrm{s}}^{2}} \frac{4}{\pi \Delta s}-\frac{3 \hat{k}_{\mathrm{p}}^{4}-4 \hat{k}_{\mathrm{p}}^{2} \hat{k}_{\mathrm{s}}^{2}+3 \hat{k}_{\mathrm{s}}^{4} \frac{i \Delta s}{8}+\frac{\Delta s}{4 \pi \hat{k}_{\mathrm{s}}^{2}}\left[3 \hat{k}_{\mathrm{p}}^{4} \log \frac{\hat{k}_{\mathrm{p}} \Delta s}{4}-4 \hat{k}_{\mathrm{p}}^{2} \hat{k}_{\mathrm{s}}^{2} \log \frac{\hat{k}_{\mathrm{p}} \Delta s}{4}+3 \hat{k}_{\mathrm{s}}^{4} \log \frac{\hat{k}_{\mathrm{s}} \Delta s}{4}+2 \hat{k}_{\mathrm{s}}^{4} \log \frac{\hat{k}_{\mathrm{p}}}{\hat{k}_{s}}\right.}{} \\
& \left.+\hat{k}_{\mathrm{p}}^{4}\left(3 C-\frac{5}{4}\right)+\hat{k}_{\mathrm{p}}^{2} \hat{k}_{\mathrm{s}}^{2}(4 C+2)+\hat{k}_{\mathrm{s}}^{4}\left(3 C-\frac{11}{4}\right)\right],
\end{aligned}
$$

$T_{n n}^{122}=T_{n n}^{221}=0 ;$

when $m \neq n$,

$$
\begin{aligned}
& T_{m n}^{121}=-\frac{i}{4} \hat{k}_{\mathrm{s}}^{2} \Delta s H_{0}^{(1)}\left(\hat{k}_{\mathrm{s}} s_{m n}\right)-\frac{i \Delta s}{\hat{k}_{\mathrm{s}}^{2}}\left[\frac{1}{s_{m n}}\left\{\hat{k}_{\mathrm{p}}^{3} H_{1}^{(1)}\left(\hat{k}_{\mathrm{p}} s_{m n}\right)-\hat{k}_{\mathrm{s}}^{3} H_{1}^{(1)}\left(\hat{k}_{\mathrm{s}} s_{m n}\right)\right\}\right. \\
& \left.+\frac{3}{s_{m n}^{2}}\left\{\hat{k}_{\mathrm{p}}^{2} H_{0}^{(1)}\left(\hat{k}_{\mathrm{p}} s_{m n}\right)-\hat{k}_{s}^{2} H_{0}^{(1)}\left(\hat{k}_{s} s_{m n}\right)\right\}-\frac{6}{s_{m n}^{3}}\left\{\hat{k}_{p} H_{1}^{(1)}\left(\hat{k}_{\mathrm{p}} s_{m n}\right)-\hat{k}_{\mathrm{s}} H_{1}^{(1)}\left(\hat{k}_{\mathrm{s}} s_{m n}\right)\right\}\right] \\
& -\frac{\hat{k}_{\mathrm{p}}^{4}+\hat{k}_{\mathrm{s}}^{4}}{4 \pi \hat{k}_{\mathrm{s}}^{2}}\left[\Delta s \log s_{m n}-\int_{s_{n}-\Delta s / 2}^{s_{n}+\Delta s / 2} \log \left|s_{m}-\hat{\zeta}_{1}\right| d \hat{\zeta}_{1}\right]-\frac{\hat{k}_{\mathrm{p}}^{2}-\hat{k}_{\mathrm{s}}^{2}}{\pi \hat{k}_{\mathrm{s}}^{2}}\left[\frac{\Delta s}{s_{m n}^{2}}-\int_{s_{n}-\Delta s / 2}^{s_{n}+\Delta s / 2} \frac{d \hat{\zeta}_{1}}{\left(s_{m}-\hat{\zeta}_{1}\right)^{2}}\right], \\
& T_{m n}^{222}=-\frac{i}{4} \hat{k}_{s}^{2}\left(1-2 \frac{\hat{k}_{p}^{2}}{\hat{k}_{s}^{2}}\right)^{2} \Delta s H_{0}^{(1)}\left(\hat{k}_{p} s_{m n}\right)-\frac{i \Delta s}{\hat{k}_{s}^{2}}\left[\frac{\hat{k}_{s}^{2}}{s_{m n}}\left\{\hat{k}_{p}\left(1-2 \frac{\hat{k}_{p}^{2}}{\hat{k}_{s}^{2}}\right) H_{1}^{(1)}\left(\hat{k}_{p} s_{m n}\right)+\hat{k}_{s} H_{1}^{(1)}\left(\hat{k}_{s} s_{m n}\right)\right\}\right. \\
& \left.-\frac{3}{s_{m n}^{2}}\left\{\hat{k}_{p}^{2} H_{0}^{(1)}\left(\hat{k}_{p} s_{m n}\right)-\hat{k}_{s}^{2} H_{0}^{(1)}\left(\hat{k}_{s} s_{m n}\right)\right\}+\frac{6}{s_{m n}^{3}}\left\{\hat{k}_{p} H_{1}^{(1)}\left(\hat{k}_{p} s_{m n}\right)-\hat{k}_{s} H_{1}^{(1)}\left(\hat{k}_{s} s_{m n}\right)\right\}\right] \\
& -\frac{3 \hat{k}_{p}^{4}-4 \hat{k}_{p}^{2} \hat{k}_{s}^{2}+3 \hat{k}_{s}^{4}}{4 \pi \hat{k}_{s}^{2}}\left[\Delta s \log s_{m n}-\int_{s_{n}-\Delta s / 2}^{s_{n}+\Delta s / 2} \log \left|s_{m}-\hat{\zeta}_{1}\right| d \hat{\zeta}_{1}\right]-\frac{\hat{k}_{p}^{2}-\hat{k}_{s}^{2}}{\pi \hat{k}_{s}^{2}}\left[\frac{\Delta s}{s_{m n}^{2}}-\int_{s_{n}-\Delta s / 2}^{s_{n}+\Delta s / 2} \frac{d \hat{\zeta}_{1}}{\left(s_{m}-\hat{\zeta}_{1}\right)^{2}}\right] \text {, }
\end{aligned}
$$

$T_{m n}^{122}=T_{m n}^{221}=0$

where $H_{1}^{(1)}(\cdot)$ is the first-order Hankel function of the first kind; $\hat{k}_{s}=a k_{s}$ is the normalized $S$-wave number in the elastic background medium; $C \approx 0.5772$ is Euler's constant; $s_{n}$ and $s_{m}$ are equal to $-1+n \Delta s$ and $-1+m \Delta s$, respectively; and $s_{m n}=\left|s_{m}-s_{n}\right|$. Note that eqs (B3) and (B6) mean

$T_{122}\left(x_{1}, 0 \mid \zeta_{1}, 0\right)=T_{221}\left(x_{1}, 0 \mid \zeta_{1}, 0\right)=0$. 